\title{
A Pipeline for the Generation of Realistic 3D Synthetic Echocardiographic Sequences: Methodology and Open-access Database.
}

\author{
M. Alessandrini, M. De Craene, O. Bernard, S. Giffard-Roisin, P. Allain, I. Waechter-Stehle, J. Weese, E. Saloux, \\ H. Delingette, M. Sermesant and J. D'hooge
}

\begin{abstract}
Quantification of cardiac deformation and strain with 3D ultrasound takes considerable research efforts. Nevertheless, a widespread use of these techniques in clinical practice is still held back due to the lack of a solid verification process to quantify and compare performance. In this context, the use of fully synthetic sequences has become an established tool for initial in silico evaluation. Nevertheless, the realism of existing simulation techniques is still too limited to represent reliable benchmarking data. Moreover, the fact that different centers typically make use of in-house developed simulation pipelines makes a fair comparison difficult.

In this context, this paper introduces a novel pipeline for the generation of synthetic 3D cardiac ultrasound image sequences. State-of-the art solutions in the fields of electromechanical modeling and ultrasound simulation are combined within an original framework that exploits a real ultrasound recording to learn and simulate realistic speckle textures. The simulated images show typical artifacts that make motion tracking in ultrasound challenging. The ground-truth displacement field is available voxelwise and is fully controlled by the electromechanical model. By progressively modifying mechanical and ultrasound parameters, the sensitivity of 3D strain algorithms to pathology and image properties can be evaluated.

The proposed pipeline is used to generate an initial library of 8 sequences including healthy and pathological cases, which is made freely accessible to the research community via our project web-page $\rrbracket$.
\end{abstract}

Index Terms-3D echocardiography, cardiac strain, motion estimation, evaluation, synthetic sequences, electromechanical model, ultrasound simulation.

\section{INTRODUCTION}

Optimal treatment of cardiac disease requires early detection of abnormalities and accurate monitoring tools. Echocardiography remains the modality of choice for this purpose given it is safe (i.e. it does not make use of ionizing radiation), cheap

Martino Alessandrini and Jan D'hooge are with the Department Cardiovascular Sciences, Laboratory of Cardiovascular Imaging and Dynamics, Catholic University of Leuven (KU Leuven), Leuven, Belgium.

Mathieu De Craene and Pascal Allain are with Philips Research, Medisys, Suresnes, France

Olivier Bernard is with Université de Lyon; CREATIS; CNRS UMR5220; Inserm U1044; INSA-Lyon; Université Lyon 1; Lyon 69622, France.

S. Giffard-Roisin, Maxime Sermesant and Hervé Delingette are with the Inria-Asclepios Project, Sophia Antipolis, France.

Juergen Wesse and Irina Waechter-Stehle are with Philips Research Laboratories, Hamburg, Germany.

Eric. Saloux is with the Department of cardiology, CHU Caen, France.

Copyright (c) 2010 IEEE. Personal use of this material is permitted.

However, permission to use this material for any other purposes must be obtained from the IEEE by sending a request to pubs-permissions@iee.org. compared to other imaging modalities and portable (i.e. can easily be used bed-side). Most importantly, echocardiography operates at high temporal resolution and is thus naturally suited to study heart dynamics. During the last decade, the introduction of 2D matrix array transducer technology has made volumetric (3D) ultrasound imaging (RT3DUS) in clinical routine feasible [1], [2].

Despite these important advances in ultrasound technology, assessment of cardiac function is generally limited to global measures (e.g. ejection fraction, stroke volume, cardiac output). Nevertheless, the analysis of cardiac motion and deformation/strain at a regional level also contains important clinical information: e.g. regional function impairment in the case of ischemia or cardiomyopathy [3]. Moreover, the comparison of the timing of segmental strain curves can help detect dyssynchrony [4]. Currently, this information relies on the visual interpretation of regional wall motion and deformation and is therefore subjective and not strongly reproducible between observers. For this reason, different approaches towards the quantification of regional heart function with RT3DUS have been proposed, some of which have recently become commercially available [5].

Obviously, the introduction of a new technology in clinical diagnosis requires a solid verification process of the proposed methodology. For ultrasound quantification of heart motion the following approaches are usually adopted:

- in vivo in human subjects by using a different imaging modality (e.g. tagged magnetic resonance imaging) as reference method [6], [7]. Unfortunately these reference techniques have their own limitations and inaccuracies. Additionally, a direct comparison requires spatiotemporal co-registration of the two datasets which is challenging and introduces inaccuracies itself.

- in vivo in open-thorax animal preparations by using sonomicrometry as gold standard reference technique [8][10]. Unfortunately, the employment of sonomicrometry implies that reference values are only available in 1 or 2 segments of the left ventricle, i.e. where the piezoelectric crystals are located, and image quality is typically superior (i.e. not representative) to what is encountered in clinical routine. Moreover, piezoelectric crystals return stronger echoes than cardiac tissue and appear as brighter spots in the ultrasound images. This, in principle, can ease (and thus bias) the tracking problem. Finally, this set-up should be used with moderation given the ethical 


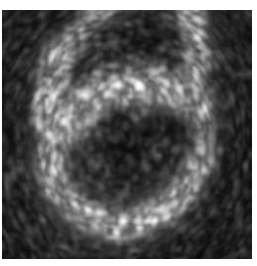

(a)

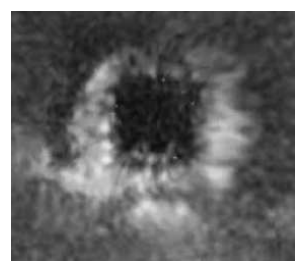

(b)

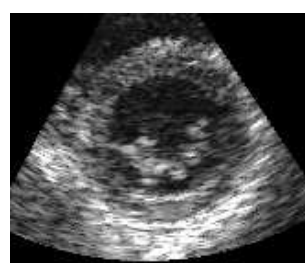

(c)
Fig. 1. Synthetic echocardiographic images used in [19](a), [20](b) and [21](c). All techniques present intrinsic limitations which limit their usability as benchmarking tools. (a) employs a simplistic speckle model (i.e. high speckle intensity inside the muscle and low intensity outside) leading to unrealistic binary-like images. In (b) the simulated cine-loop is obtained by warping the B-mode pixel images of a real recording, thus producing texture warping artifacts. In (c) the presence of warping artifacts is avoided by using an ultrasound simulator. Yet, the reference motion field is computed by applying an elastic registration technique to the real recording working as template and therefore $i$ ) no control on the simulated motion is possible, ii) errors of the registration algorithm are directly reflected on the simulated motion and, moreover, iii) the benchmark displacement is biased towards the elastic registration technique employed. The pipeline described in this paper overcomes such limitations.

implications and is in addition expensive;

- in vitro by using a physical cardiac phantom in which motion and deformation can be mechanically controlled [11], [12]. As above, sonomicrometry is used as reference and image quality is not representative for data taken in clinical routine. In addition, mimicking cardiac motion in a mock model can be challenging. In this context it is worth mentioning that, in order to attain more realistic simulators, in recent years mock systems able to host $e x$ vivo part of or entire explanted hearts, mainly of swines, were successfully developed [13]-[15];

- in silico by using computer generated sequences of echocardiographic images [16]-[19]. In this case no intermodal registration is needed (unlike tagged MRI) and the exact motion/deformation is known at each point (voxel) inside the myocardium (unlike sonomicrometry). Additionally digital data can be easily accessed by the research community thus eliminating the need for complex experimental setups. Unfortunately, the level of realism of existing simulated sequences is still too limited to represent reliable reference data.

\section{A. State-of-the-art in cardiac ultrasound simulation}

Existing simulation techniques mostly combine two elements: one model for anatomy and motion of the myocardium and one ultrasound simulator to mimic the image formation process. In this context considerable efforts have been spent in order to have the most realistic models for anatomy and motion and the most physically sound and computationally effective ultrasound simulations.

Preliminary work making use of synthetic cardiac US sequences include the ones in [24] and [25], where an annular shape was adopted to represent a LV short-axis cross section in combination with a radial motion model to account for contraction and expansion. Since the introduction of 3D scanners, modeling the whole muscle in $3 \mathrm{D}$ has received considerable attention. For example, a truncated prolate spheroid was employed in [16] to model the LV shape in 3D with a complex kinematic model to rule the motion pattern. In [17] a truncated ellipsoid was adopted for both ventricles to which an electromechanical model of cardiac motion was applied [26]. Finally, in [19], the geometry was obtained through segmentation of cine MR images, while the Bestel-ClementSorine (BCS) electromechanical model [22] was used for computing deformation through the cardiac cycle. As compared to kinematic models, electromechanical ones have the benefit of directly relating the contraction law with its biophysical causes which allows for a more realistic incorporation of physiological and pathological conditions.

Regarding ultrasound simulators, FieldII [27], [28] is commonly considered as the state-of-the-art for linear acoustics: it provides a library for the calculation of pressure fields from arbitrarily shaped and apodized transducers. Nevertheless the computational burden associated to FieldII makes it unfeasible for simulating large data-sets especially in 3D. In this context COLE [23] was recently introduced as a fast alternative for generating 3D ultrasound sequences: it accelerates the convolution of a $3 \mathrm{D}$ point spread function by multiple 1D convolutions while allowing the integration of various simulated or measured beam profiles as a lookup table.

Ultrasound simulators typically model the tissue response as a collection of point scattering centers. To account for the different acoustic impedance between the myocardium and the blood pool all aforementioned frameworks proceed in the following binary fashion: high amplitude is assigned to scatterers inside the muscle and a low amplitude to the ones outside. As a consequence, the level of realism of the resulting ultrasound images remains highly unsatisfactory: surrounding structures such as papillary muscles and heart valves are neglected as well as typical artifacts such as reverberations, clutter noise, signal dropout and local intensity variations due to changing cardiac fiber orientation (see Fig. 1 a)). All these elements are critical as they have a major impact on the performance of algorithms for motion/deformation estimation.

Two recent solutions represented a considerable leap forward in this scenario [20], [21]. In those works, instead of a simple binary mask, a real ultrasound recording was used as a template to obtain realistic speckle textures. By doing so, surrounding structures and ultrasound artifacts were directly transferred from the template to the simulation (cf. Fig. 1 (b) and (c)). However, both techniques suffer from intrinsic limitations. In [20] the synthetic sequence was obtained by warping the B-mode pixel data of the template acquisition according to the benchmark motion field obtained from a BCS model [22]. Such an approach unavoidably introduced unrealistic warping artifacts in the simulated speckle texture (cf. Fig. 1(b)). In particular, the framework enforced an unrealistically high temporal speckle correlation which could mislead the performance assessment of speckle tracking algorithms. To avoid this, in [21] an ultrasound simulator (FieldII) was used to handle the generation of the synthetic images independently. Hereto, the template sequence was used to compute the amplitude distribution of the scatter map fed to the ultrasound simulator. As compared to [20], the approach allowed generating considerably more realistic speckle images 


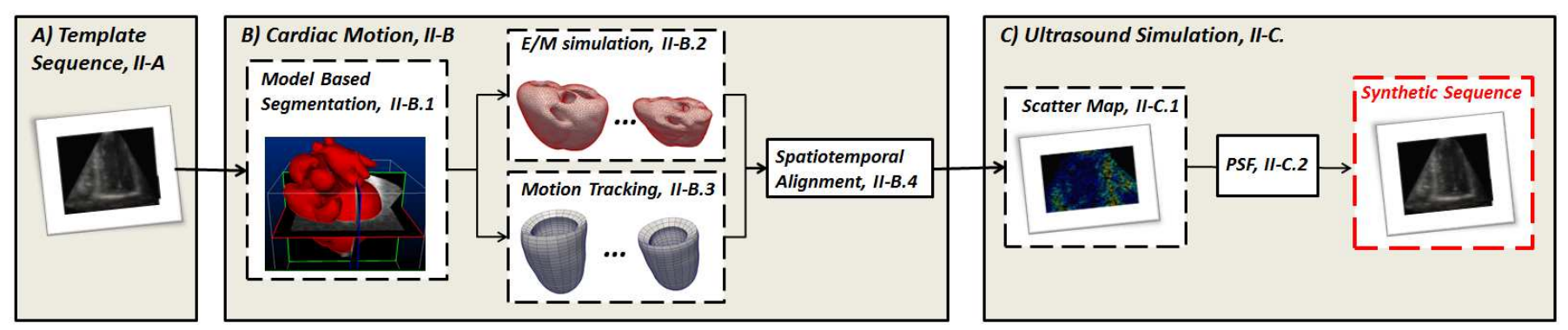

Fig. 2. Proposed pipeline for the simulation of ultra realistic cardiac ultrasound sequences: (A) a clinical recording works as a template for speckle texture and anatomy definition; (B) an electromechanical model controls the synthetic heart motion [22]; (C) an ultrasound simulation environment merging information from the template image sequence and the electromechanical model accounts for the image formation process [23]. In the simulated sequence the cardiac motion is fully controlled by the electromechanical model while the visual appearance is very similar to the one of a real acquisition. Each block specifies the number of the section where its description is detailed.

(cf. Fig. 1(c)). The synthetic motion was then obtained by applying the motion field estimated from the template sequence using the spatio-temporal elastic registration technique in [29]. This represented a main limitation since $i$ ) no control on the simulated motion was possible, $i$ i) motion estimation errors in the template sequence were directly reflected on the simulated motion and iii) the benchmark displacement/deformation was naturally biased towards the registration technique employed in [29] and hence unsuited to benchmark generic motion estimation algorithms. Moreover, the approach in [21] was proposed only for $2 \mathrm{D}$ ultrasound.

\section{B. Contribution of the paper}

This paper builds on our previous recent contributions [19][21] and introduces a novel framework for the generation of 3D realistic synthetic echocardiographic sequences overcoming the limitations of existing approaches. The proposed simulation pipeline consists of three elements as illustrated in Fig. 2

A) A real clinical recording provided both realistic texture and geometries for the ventricles. As in [20], [21], embedding of a real image sequence improves the realism of the generated images, yielding synthetic data that fairly reproduce the challenges inherent to 3D ultrasound image and signal processing.

B) As in [19], [20], the synthetic motion field was obtained by the BCS electromechanical (E/M) model [22]. The E/M model provides the reference for heart motion and derived quantities and generates a continuous range of pathological patterns by progressively altering the parameter settings related e.g. to contraction or conduction of myocardial tissue.

C) As in [19], the fast US simulation environment COLE [23] was used to model the image formation, allowing full control over the transducer's parameters and ensuring realistic speckle patterns.

Thanks to B) the reference displacement, unlike [21], is unbiased to any motion estimation algorithm. Moreover, thanks to C) and unlike [20], motion and ultrasound simulations are decoupled and hence artifacts due to texture warping are avoided.
The proposed pipeline was used to generate a library of 8 benchmark sequences including physiological and pathological geometries and contraction patterns, i.e. ischemia and dyssynchrony. The generated sequences along with the reference displacements are made freely available to the research community via an open-access database 2 . With this common database, we aim to $i$ ) enable a more thorough and reliable comparison of existing techniques for motion/deformation assessment from 3D US ii) make the evaluation of new techniques less dependent on the specific data-set used and iii) foster more coordinated research efforts on the topic and avoid multiplication of efforts.

This paper extends the conference paper in [30]. With respect to that work the simulation framework is more detailed; simulations were extended to more cases and parameters were tuned to match physiological ranges; both image properties and mechanical properties of the generated sequences are better evaluated and commented upon.

The paper proceeds as follows. Sect. In describes the simulation pipeline. In Sect. IIII the visual realism of the created sequences is assessed qualitatively and quantitatively and the mechanical properties of the synthetic dataset are analyzed. Concluding remarks are left to Sect. IV

\section{Proposed Simulation framework}

A schematic of the pipeline is summarized in Fig. 2. Each block specifies the number of the section where it is described in detail. Briefly, a real 3D recording is used as a template for speckle texture. The first step is to segment the right (RV) and left ventricles (LV) on the first frame of the $3 \mathrm{D}$ template sequence (Sect. (II-A). The E/M model is then applied to the segmented 3D geometry to simulate one cardiac cycle (Sect. III-B2). The E/M model is used to displace a 3D cloud of point scatterers mimicking tissue echogenicity. To achieve realistic speckle texture, scattering amplitude is sampled from the template recording Sect. [II-C1] This requires aligning the simulation and template recordings in space and time (Sect. II-B4). Hereto, 3D LV tracking of the template is used for spatial matching. Namely, this generates a set of spatial landmarks that are used for canceling the motion of the template image sequence (Sect. [I-B3). The scatter map is then

${ }^{2}$ https://team.inria.fr/asclepios/data/straus/ 
fed to the ultrasound simulator to generate the synthetic images (Sect. II-C2). As such, the pipeline will generate synthetic sequences with fully controlled cardiac motion and ultrasound speckle texture visually similar to the one of the template recording.

\section{A. Template image sequences}

A 3D ultrasound sequence is used as a template sequence for defining the geometry and providing the texture of the ultrasound simulator. In this paper, two 3D ultrasound sequences (one full cycle from end of diastole, ED) were acquired from an apical view using a Philips iE33 ultrasound machine with a matrix array transducer (X5). The first sequence (image size $224 \times 176 \times 208$ voxel $^{3}$, voxel size $0.7 \times 0.9 \times 0.6 \mathrm{~mm}^{3}$, frame rate $23 \mathrm{~Hz}$ ) was acquired from a healthy volunteer. The second sequence (image size $224 \times 176 \times 208$ voxel $^{3}$, voxel size $0.7 \times 0.9 \times 0.6 \mathrm{~mm}^{3}$, frame rate $21 \mathrm{~Hz}$ ) corresponded to a dilated left ventricle (DLV) and was acquired from a dyssynchronous patient candidate to a cardiac resynchronization therapy.

The ultrasound machine settings were optimized to obtain the best image quality and the optimal visualization of the full LV myocardium from the apex to the base. To optimize frame rate we used a four subvolumes acquisition mode and the field of view was limited to the LV cavity. To have similar temporal resolution in the two sequences, healthy and dilated cases were acquired with the same field of view.

\section{B. Cardiac Motion}

1) Geometry: The first frame of each template sequence was segmented automatically. Hereto, the model-based technique for full heart segmentation in [31], initially developed for CT and recently extended to ultrasound [32], [33], was used (cf. Fig. 2).

2) Electromechanical simulation: From the segmentation result the left and right ventricular geometries were extracted. The right ventricle was first extruded along the mesh normals to produce a RV epicardial surface, not returned by the segmentation software [31]. Extrusion was controlled in order to have a physiologic thickness of $\sim 4.5 \mathrm{~mm}$ of the RV free wall [34]. From this surface mesh a volumetric tetrahedral mesh was then obtained [35] and passed as input to the motion simulator. The latter applies the Bestel-Clement-Sorine E/M model [22] and was implemented in the SOFA frameworks. This model was chosen for its realistic properties. It is based on energy-preserving equations, includes the Frank-Starling effect and complies with the four cardiac phases (isovolumic contraction, ejection, passive and active filling). This model showed good predictive power [36] in the context of cardiac resynchronization therapy. Moreover, tests on preload, afterload and inotropy as well as a preliminary specificity study performed in [22] proved its good physiological behavior and its ability to simulate healthy and pathological cases.

By varying the values of the parameters of the E/M simulator a library of cases was created including healthy and pathological conditions. In particular:

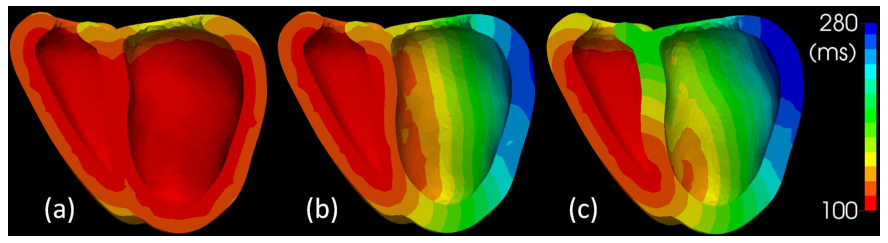

Fig. 4. Depolarization times for the LBBB simulations. (a) For the normal simulation, both LV and RV endocardium are activated simultaneously, resulting in a synchronous activation. (b) For LBBBsmall, only the RV endocardium is early activated. The septum still gets activated through the RV. (c) For the LBBB simulation, only the endocardial RV free wall is first activated. This delays activation in the septum (particularly in the base) and the lateral wall.

- From the normal geometry one mechanical simulation of a healthy heart was generated by assigning normal contractility and stiffness values to all heart segments, as defined by the standard American Heart Association (AHA) 17 segments model [37]. In particular, mechanical parameters were tuned in order to match ejection fraction (EF) measured on the corresponding template acquisition (cf. Fig. 5(a));

- From the healthy geometry, four ischemic simulations were generated by altering contractility and stiffness in diseased segments. Hereto, two types of segments were considered: segments corresponding to the area at risk (Isch+) were considered as fully ischemic and had contraction parameters altered more significantly, while adjacent segments (Isch) were considered as mildly ischemic. Remote segments were assigned normal values. The four ischemic simulations corresponded to: a distal and proximal occlusion of the Left Anterior Descending artery (LADdist and LADprox respectively); occlusions of Right Coronary Artery (RCA) and Left Circumflex (LCX). Diseased segments for each case are illustrated in Fig. 3 .

- From the dilated geometry 3 simulations were generated: one synchronous (sync) and two dyssynchronous. Dyssynchrony, as induced by Left Bundle Branch Block (LBBB), was modeled by progressively delaying LV activation with respect to RV. First, for generating the sync simulation on the dilated geometry, both LV and RV endocardial surfaces were early activated simultaneously. This induces a fast activation of both the LV and RV and results in a globally synchronous contraction pattern. Mechanical parameters were tuned for the sync sequence in order to match EF of the template DLV acquisition (cf. Fig. 5(b)). They were then left unchanged for the dyssynchronous simulations. For generating the first case of dyssynchrony (LBBBsmall) we activated RV early. We also generated a more severe case of dyssynchrony (LBBB) by activating only a patch of the RV free wall. Colormaps of the depolarization times for the three cases are plotted in Fig. 4. These different activation patterns cause a progressive delay of LV contraction with respect to the RV, especially in the LV lateral wall.

The values of the relevant mechanical parameters for healthy, ischemic and DLV simulations are provided in Ap- 


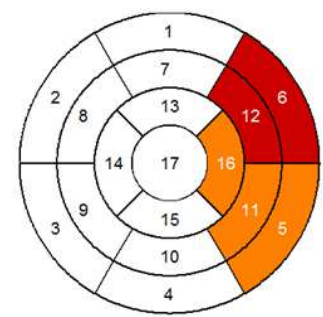

(a) LCX

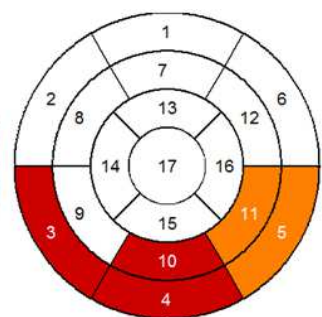

(b) RCA

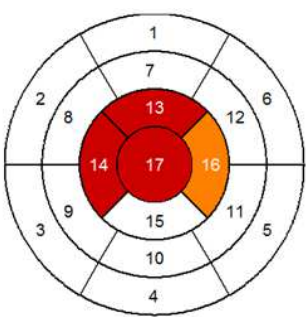

(c) LAD distal

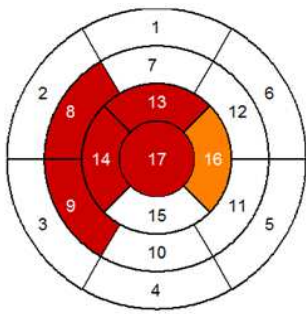

(d) LAD proximal

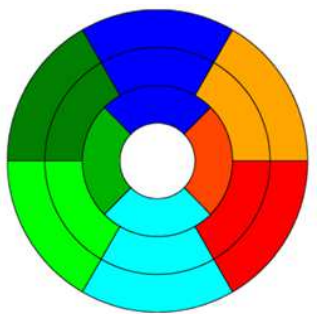

(e) color notation

Fig. 3. (a,b,c,d): Segments involved in the simulations of ischemia. Red and orange denote severe and mild ischemic segments respectively. (e) Color notation used in the results section.

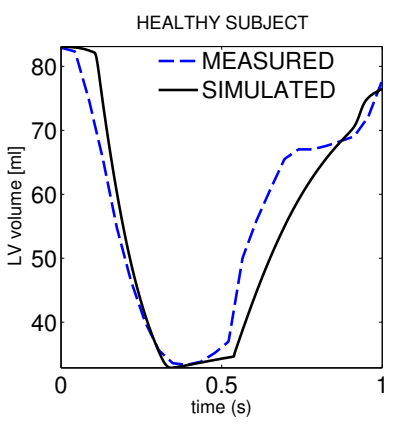

(a)

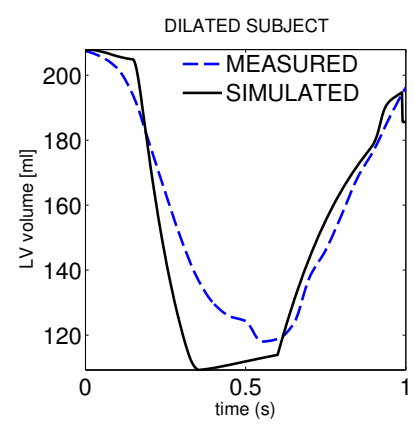

(b)
Fig. 5. Comparison between LV volumes of the E/M simulation and measured on the template recording for healthy subject (a) and DLV (b). $\mathrm{EF}_{\text {measured }}=$ $\mathrm{EF}_{\text {simulated }}=60 \%$ in (a) and $\mathrm{EF}_{\text {measured }}=43 \%, \mathrm{EF}_{\text {simulated }}=47 \%$ in (b).

pendix A The resulting 8 simulated sequences constitute the benchmarking dataset made available open-access. Their features are summarized in Table \.

TABLE I

OPEN-ACCESS DATABASE.

\begin{tabular}{|c|c|c|c|}
\hline Simulation & $\begin{array}{c}\text { Geometry } \\
\text { normal/dilated }\end{array}$ & $\begin{array}{c}\text { Contractility } \\
\text { normal/ischemia }\end{array}$ & $\begin{array}{l}\text { Activation } \\
\text { sync/async } \\
\end{array}$ \\
\hline 1. Healthy & $\checkmark$ & $\checkmark$ & $\checkmark$ \\
\hline \multicolumn{4}{|l|}{ Ischemic: } \\
\hline $\begin{array}{l}\text { 2. LADdist } \\
\text { 3. LADprox } \\
\text { 4. LCX } \\
\text { 5. RCA }\end{array}$ & $\checkmark$ & $\checkmark$ & $\checkmark$ \\
\hline \multicolumn{4}{|l|}{ DLV: } \\
\hline $\begin{array}{l}\text { 6. Sync } \\
\text { 7. LBBB } \\
\text { 8. LBBBsmall }\end{array}$ & $\checkmark$ & $\checkmark$ & $\checkmark$ \\
\hline
\end{tabular}

3) Motion Tracking: In parallel to the E/M simulation, from the same segmentation result of [31] endo- and epicardial borders of the left ventricle (LV) were extracted and converted to the QLab software (3DQ Advanced plugin) [38] format. They were slightly edited to fit image borders by an experienced cardiologist (E. S.). The contours were then propagated to the entire sequence by a sparse implementation of the Demons algorithm [39] and then further edited in 4D to ensure a smooth propagation of manual updates. The sparse Demons algorithm [39] was selected given the good tracking accuracy, competitive with the best performing algorithms considered in the recent comparison study in [19], and the low computational cost $(\sim 0.5 \mathrm{~s} /$ frame $)$. The result was a set of landmarks over time sampling the LV endo- and epicardial surfaces regularly in the circumferential radial and longitudinal directions. These landmarks will be used for canceling the motion of the template image sequence when generating the texture of the ultrasound simulation (cf. Sect. II-B4).

4) Spatiotemporal Alignment: The simulated heart motion is completely independent of the one of the template recording. Since, in the proposed framework, speckle intensity is sampled from the real acquisition, alignment of the two datasets is therefore needed to establish from which frame to sample (temporal alignment) and in which position (spatial alignment).

Temporal alignment is performed by linearly stretching/shrinking the time axis of the template recording in order to match relevant cardiac events used as temporal landmarks. In this paper, opening and closure of the mitral and the aortic valves, together with the beginning and the end of the sequence (ED) were chosen as landmarks. Namely, given a generic simulation time $k_{\text {sim }}$, temporal synchronization identifies the matched time instant $t_{\text {templ }}\left(k_{\text {sim }}\right)$ on the template recording computed as:

$$
t_{t e m p l}\left(k_{\text {sim }}\right)=\frac{k_{\text {sim }}-k_{\text {sim }}^{n}}{k_{\text {sim }}^{n+1}-k_{\text {sim }}^{n}}\left(k_{\text {templ }}^{n+1}-k_{\text {templ }}^{n}\right)+k_{\text {templ }}^{n} .
$$

being $k_{\text {sim }}^{n}$ and $k_{\text {templ }}^{n}$ the $n$-th temporal landmark on the E/M simulation and the template recording respectively and $k_{\text {sim }}^{n} \leq$ $k_{\text {sim }} \leq k_{\text {sim }}^{n+1}$. As $t_{\text {templ }}\left(k_{\text {sim }}\right)$ is in general non-integer valued, it is used to identify the two closest image frames $k_{\text {templ }}=\left\lfloor t_{\text {templ }}\left(k_{\text {sim }}\right)\right\rfloor$ and $k_{\text {templ }}+1=\left\lceil t_{\text {templ }}\left(k_{\text {sim }}\right)\right\rceil$. The two frames will serve as template for speckle intensity as explained in Sect. II-C1.

After synchronization, spatial alignment is needed to align the E/M geometry with the previously selected (i.e. matched in time) image frame. Namely, given an arbitrary point $\mathbf{x}$ in the simulation space at time $k_{\text {sim }}$, we seek for a dense transformation $\mathcal{T}_{k_{\text {sim }} \rightarrow k_{\text {tem } p l}}(\mathbf{x})$ returning its matched position (i.e. in the same anatomical location) in the template frame $I_{k_{t e m p l}}$. The process is identical for $I_{k_{t e m p l}+1}$ and is omitted for clarity.

The transformation is computed by matching the position of 


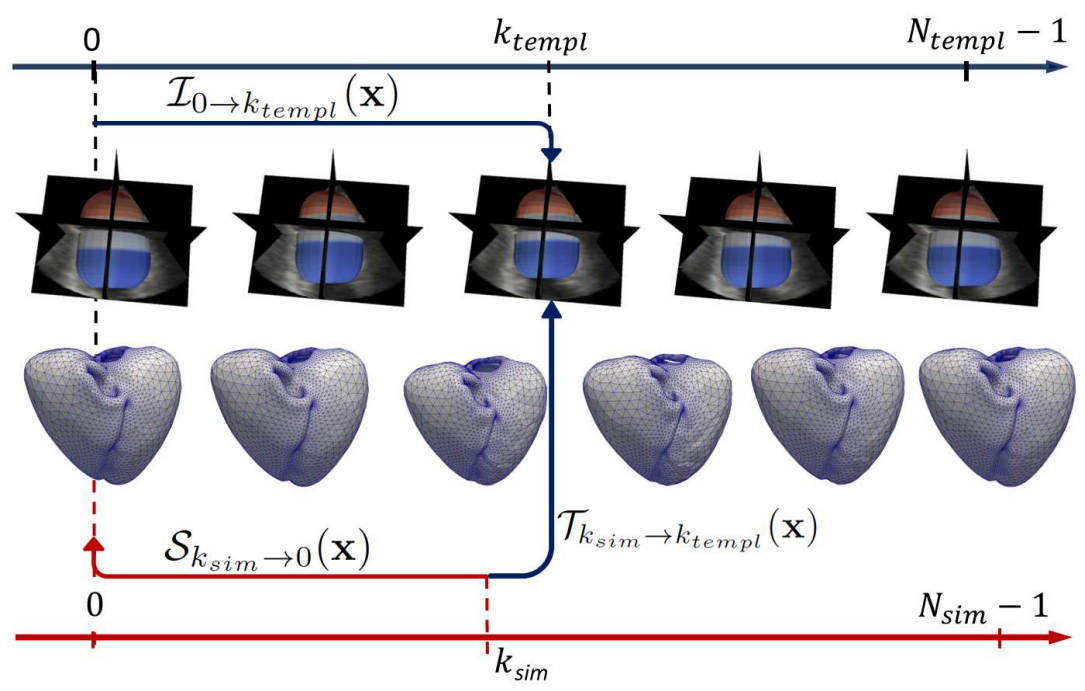

Fig. 6. Spatio-temporal mapping used to estimate the echogenicity of a background scatterer. The top row reports the template sequence along with the associated set of segmentation meshes obtained with QLab. The bottom row reports the sequence of simulation meshes obtained with the BCS E/M model. The two time axis of the template acquisition and of the simulation are illustrated in blue and red respectively. Temporal matching (cf. (1) assigns to the simulation mesh at time $k_{\text {sim }}$ a frame $k_{\text {templ }}$ in the template acquisition. Spatial matching then aligns the myocardial geometries in the two selected frames. The two used transformations are illustrated using bent arrows.

the myocardium in the simulation at time $k_{\text {sim }}$ (defined by the $\mathrm{E} / \mathrm{M}$ simulation mesh), with the position of the myocardium in the template frame $k_{t e m p l}$ (given by the semiautomatic segmentation of the template frame $k_{t e m p l}$, Sect. [I-A). The global transformation $\mathcal{T}_{k_{s i m} \rightarrow k_{\text {templ }}}(\mathbf{x})$ was split in two terms: one backward transformation $\mathcal{S}_{k_{s i m} \rightarrow 0}(\mathbf{x})$ in the simulation space and one forward transformation in the template space $\mathcal{I}_{0 \rightarrow k_{\text {templ }}}(\mathbf{x})$. Namely, $\mathcal{S}_{k_{\text {sim }} \rightarrow 0}(\mathbf{x})$ brings a point $\mathbf{x}$ in the simulation space back to its position at time 0 ; then, the transformation $\mathcal{I}_{0 \rightarrow k_{t e m p l}}(\mathbf{x})$ brings it forward in time to its matched position in the image domain. Both mappings are illustrated on Fig. 6. The composition of these mappings give the desired transformation:

$$
\mathcal{T}_{k_{\text {sim }} \rightarrow k_{\text {templ }}}=\mathcal{I}_{0 \rightarrow k_{\text {templ }}} \circ \mathcal{S}_{k_{\text {sim }} \rightarrow 0} .
$$

Such a splitting of $\mathcal{T}$ is correct since, in the proposed framework, simulation space and image space are aligned at the first frame. In order to avoid warping artifacts in the simulated speckle texture, spatial mapping must be smooth outside the LV myocardium (cf. Sect. II-C). For this reason, combined with the ease of implementation, thin plate splines (TPS) [40] were used to compute both mappings. The TPS transform was parametrized by the mesh nodes' positions (i.e. the E/M meshes in the case of $\mathcal{S}$ and the tracking meshes in the case of $\mathcal{I}$ ). Note that alternative regression techniques could be used instead of TPS [41]. Note also that TPS could not be applied to compute the global transformation $\mathcal{T}$ directly given that image and simulation meshes have in general different properties (nodes and faces) since they are obtained in different ways.

\section{Ultrasound simulation}

The first step in the ultrasound simulation is the definition, at each simulation time $k_{\text {sim }}$, of a 3D cloud of point scatterers mimicking the local tissue echogenicity. Each point scatterer is assigned a scattering amplitude related to the amount of energy reflected back to the probe (i.e. the intensity of the echo signal). Our approach consists in moving the scatterers according to the E/M simulation while sampling their amplitude from the real template recording. This will generate synthetic sequences with fully controlled cardiac motion and realistic ultrasound speckle texture. This procedure exploits all steps previously described and is the core of the proposed framework. Its description is addressed in Sect. II-C1. The scatter map is then fed to the US simulator to generate the synthetic images (Sect. II-C2).

1) Scatter map: We call $\mathbf{x}_{i}^{k_{s i m}}=\left[x_{i}^{k_{s i m}}, y_{i}^{k_{s i m}}, z_{i}^{k_{s i m}}\right]^{T}$ and $a_{i}^{k_{s i m}}$ the position and the amplitude of the $i^{\text {th }}$ scatterer at time $k_{\text {sim }}$. We call $I_{j}(\mathbf{x}) \in[0,255]$ the intensity of the $j$-th template frame at position $\mathbf{x}$. Note that when non-integer spatial positions need to be accessed, the intensity value is obtained by using bi-cubic interpolation.

The first step is to generate a scatter map for the first frame $k_{\text {sim }}=0$. The coordinates of the scatterers are obtained by uniformly sampling $N_{\text {scatt }}$ points $\mathbf{x}_{i}^{0}$ through the image domain. Their echogenicity is obtained by sampling the intensity of the template frame, namely $a_{i}^{0}=F\left(I_{0}\left(\mathbf{x}_{i}^{0}\right)\right)$, where $F$ is the non linear transformation applied to the intensity value in order to compensate for the log-compression traditionally performed in the ultrasound device prior to display. In particular, $F$ is defined in such a way that:

$$
20 \log _{10}[F(I(\mathbf{x}))]+d B=d B \cdot \frac{I(\mathbf{x})}{255},
$$

where $d B$ is the desired contrast in decibel in the simulated image.

Given $\mathbf{x}_{i}^{0}$, the first simulation mesh is used to distinguish myocardial scatterers (i.e. contained inside the mesh) from background ones (i.e. outside the mesh). We denote the number of myocardial and background scatterers as $N_{m y o}$ and 


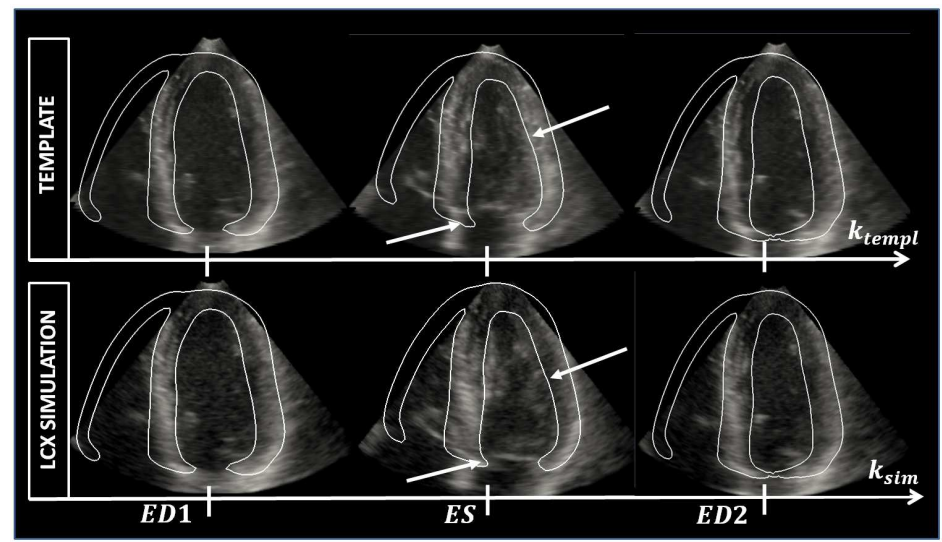

(a) A4C view

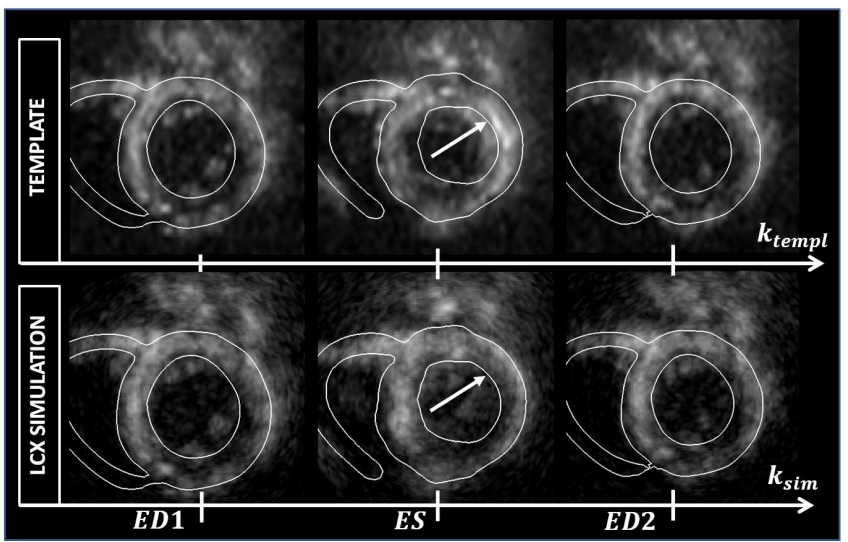

(b) SAx view

Fig. 7. Template recording (top row) and LCX simulation (bottom row) at matched time instants for an apical four chamber (a) and a short axis (b) slice. The E/M meshes (i.e. the ground truth segmentation of the LCX simulation) are superimposed to both sequences as white contours. Template, simulation and, therefore, E/M geometry are aligned by construction at time zero (i.e. at ED1). From then on, the simulation runs independently of the template recording and, therefore, the E/M geometry no longer provides a valid segmentation of the latter. In the figure, this is particularly evident at ES at the valve level in the longitudinal view and in the lateral wall on both views (cf. white arrows). This is a consequence of the fact that, as due to the simulated ischemia, contraction in the $\mathrm{E} / \mathrm{M}$ simulation is reduced as compared to the template. Videos showing the entire simulated sequences are provided at the address http://bit.ly/1vsIIhB

$N_{\text {back }}$ respectively. The barycentric coordinates of each myocardial scatterer relative to the associated mesh tetrahedron are computed and stored [42].

The scatter map generation at times $k_{\text {sim }}>0$ proceeds differently for myocardial scatterers and background ones. Myocardial scatterers move anchored to the simulation meshes to mimic the motion of cardiac tissue. This is implemented by keeping their barycentric coordinates constant over time. From the barycentric coordinates and the nodes position of the simulation mesh at the considered time $k_{\text {sim }}>0$, the absolute coordinates of myocardial scatterers $\mathbf{x}_{i}^{k_{\text {sim }}}$ are then computed [42]. As e.g. in [17], [19], [21], the amplitude of myocardial scatterers is kept constant over time, i.e. $a_{i}^{k_{s i m}}=a_{i}^{0}$. Such a choice of assigning scattering amplitude ensures having temporal coherency of the speckle texture patterns inside the myocardium. However, the underlying intensity can still vary with relative position changes over the cardiac cycle between the probe and the scatterers (see Sect. (II-B). As common in clinical scans, the apical portion of the LV epicardium was not fully visualized in the template recording (cf. Fig. 7)a)). As such, no amplitude information was available for myocardial scatterers in that region. To avoid the appearance of holes in the simulated sequences during cardiac contraction, such scatterers were assigned an artificial amplitude equal to the average of the 50 closest scatterers inside the field of view (i.e. for which amplitude information was available from the image content). This solution was found sufficient to avoid artifacts.

At each simulation time $N_{\text {back }}=N_{\text {scatt }}-N_{\text {myo }}$ background scatterers are re-drawn outside the myocardium following a uniform random distribution. Their random motion accounts for the lack of speckle coherency which is normally observed in the blood pool. Background scatterers also mimic the appearance and motion of surrounding structures as valves and papillary muscles which are not described by the electromechanical model. Background scatterers are visualized by updating their amplitude at each simulation time by using the intensity from the matched position in the template recording, as defined by the spatiotemporal mapping in Sect. II-B4 Namely, given a background scatterer $\mathbf{x}_{i}^{k_{s i m}}$ and $I_{k_{t e m p l}}^{i}$ and $I_{k_{t e m p l}+1}^{i}$ the intensities of the two consecutive template frames identified by the temporal synchronization in (1) at the matched spatial positions, i.e.

$$
I_{k_{\text {templ }}}^{i}=I_{k_{\text {templ }}}\left(\mathcal{T}_{k_{\text {sim }} \rightarrow k_{\text {templ }}}\left(\mathbf{x}_{i}^{k_{\text {sim }}}\right)\right)
$$

scattering amplitude $a_{i}^{k_{\text {sim }}}$ is computed by linearly weighting the two contributions:

$$
a_{i}^{k_{\text {sim }}}=(1-w) \cdot I_{k_{t e m p l}}^{i}+w \cdot I_{k_{t e m p l}+1}^{i}
$$

with $0<w=t_{\text {templ }}\left(k_{\text {sim }}\right)-k_{\text {templ }}<1$. The same amplitude correction in (3) is then applied. Note that the perceived motion of background structures comes from the intensity variations (5) and does not correspond to the TPS displacement field $\mathcal{T}$, which only serves to relate the two geometries. As such, while the rendered motion of surrounding structures is visually realistic, their reference displacement is not available.

2) Point Spread Function: From the 3D scatter map an ultrasound volume was simulated by convolution with the spatially variant point spread function (PSF) of the simulated imaging system. In particular COLE [23] was adopted as ultrasound simulation environment due to its good compromise between computational efficiency and accuracy [45]. The properties of the synthetic probe were set so to match as close as possible the ones of the transducer used in the acquisition of the template. Namely, the synthetic US system was sampling at $50 \mathrm{MHz}$ and equipped with a phased array transducer, which was centered at $3.3 \mathrm{MHz}$ and transmitting a Gaussian pulse with a $-6 \mathrm{~dB}$ relative bandwidth of $65 \%$. A symmetric transverse two-way beam profile was assumed, focusing at $80 \mathrm{~mm}$ when transmitting and dynamically focusing on receive. The simulated images consisted of $107 \times 80$ lines in azimuth and elevation direction over an angle of $76 \times 76$ degrees, resulting 


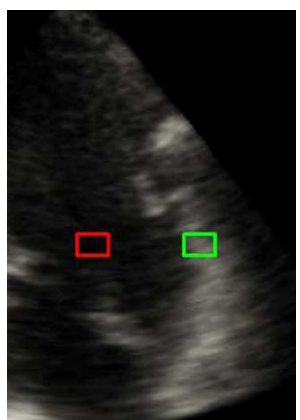

(a)

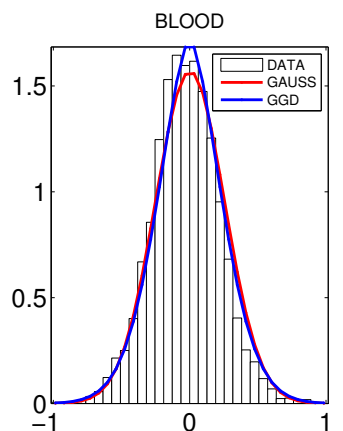

(b)

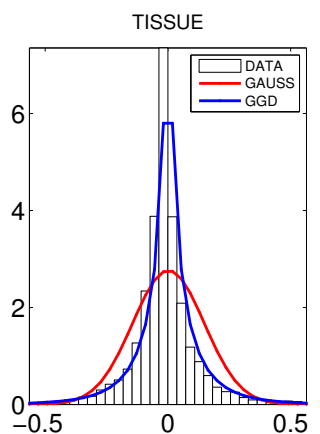

(c)

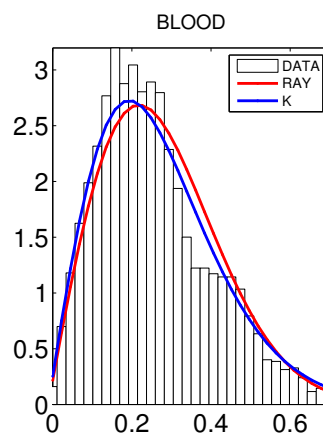

(d)

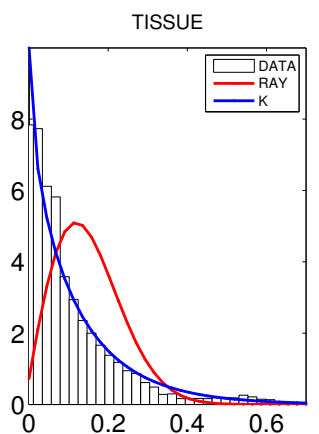

(e)

Fig. 8. Statistical distribution for two rectangular speckle patches belonging to the blood pool and the myocardium (red and green blocks in (a)). Each ROI is rectangular with side equal to three times the size of the system PSF in the corresponding direction and comprises $\sim 6800$ samples. Note that, although voxel data is reported in (a) for a more intuitive visualization, histograms were computed on raw data, i.e. before down-sampling and scan conversion. In (b) and (c) the histograms of the RF signal. In (d) and (e) the histograms for the non-compressed envelope signal. In the fitting of the RF and envelope histograms Gaussian and Rayleigh distributions show good fit respectively for fully developed speckle regions (i.e. the blood pool) while generalized Gaussian distribution (GGD) [43] and K-distribution [44] were adopted for sub-Rayleigh regions (i.e. the tissue). Consistently with what is expected on real recordings, Gaussian and Rayleigh models correctly fit the amplitude data inside the blood pool while speckle inside the muscle follows instead more heavily tailed distributions. Note that histograms have been normalized to have unitary area in order to be comparable with the associated probability density functions.

in a frame rate of $30 \mathrm{~Hz}$ assuming parallel beam forming and ECG gating. After envelope extraction, log compression and scan conversion B-mode voxel data were available.

\section{RESULTS}

\section{A. Image properties: qualitative assessment}

The proposed pipeline allows simulating ultrasound volumes visually similar to real ultrasound recordings, cf. Fig. 7 . When considering the simulated cine loop, a realistic motion for myocardium (thanks to the E/M model) and surrounding structures is observed (cf. Fig. 7). For a better dynamical perception, movies of the full simulated sequences are provided at the url http://bit.ly/1vsIIhB

The level of realism was also evaluated through a blindtest involving four expert observers: two cardiologists and two researchers (PhDs) having a technical background with expertise in analyzing and processing cardiac ultrasound datasets. The quality of single synthetic frames (i.e. of the ultrasound simulation) and of the full synthetic video sequence (i.e. of the merging between the E/M simulation and the real recording) were assessed separately. Hereto, the observers were first given a set of $14(7+7)$ images randomly extracted and shuffled from the simulated dataset and from a set of four real ultrasound recordings including the two template acquisitions (healthy and DLV) and two recordings from the recent online available database of the Challenge on Endocardial Threedimensional Ultrasound Segmentation (CETUS) [46]4. Each image displayed the 3 orthogonal slices (apical 4 chamber, apical 2 chamber and short axis) extracted from the 3D volume. Observers independently rated each image as real, synthetic or impossible to assess. The same test was repeated by distributing $4+4$ full cine loops. Observers were not aware of the ratio between simulations and real recordings. The detailed results of the blind test are reported in Appendix B. The results per class of observers are displayed in Fig. 9.

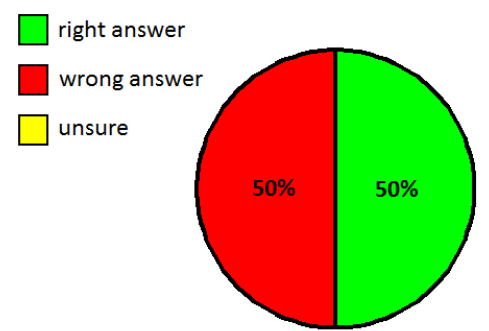

(a) clinicians - single frame $\mathrm{SP}=57 \% ; \mathrm{SE}=43 \%$

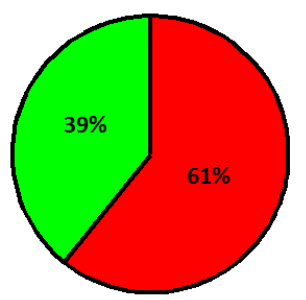

(c) non clinicians - single frame $\mathrm{SP}=36 \% ; \mathrm{SE}=43 \%$

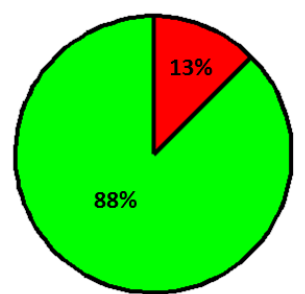

(b) clinicians - cine loop $\mathrm{SP}=75 \% ; \mathrm{SE}=100 \%$

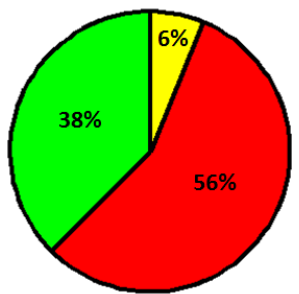

(d) non clinicians - cine loop $\mathrm{SP}=57 \% ; \mathrm{SE}=25 \%$
Fig. 9. Pie chart summarizing the results of the blind test. Specificity (SP) and sensitivity (SE) measured per class of observers are reported in the subcaptions. When still frames were displayed even expert clinicians could not tell simulations from real acquisitions (cf. charts (a) and (c)). When the cineloop was played the difference became apparent (yet, with 2 misclassified cases, cf. Table IV only for clinicians (cf. charts (b) and (d)).

Even for cardiologists, answering required attentive observation of the samples. For single frames, cardiologists admitted that it was hard to tell simulations from real acquisitions as proven by the obtained $50 \%$ error rate. Detection was instead considerably easier when videos were played. Reasons for identification of a simulated dataset were the high image quality and the sharp delineation of the endocardium. These points are further commented upon in the conclusions. For both non-clinical observers, although ratings were considerably unbalanced (cf. Fig. 9), it was difficult to detect synthetic datasets even when the cine-loop was played. 
Clearly, a statistically relevant analysis would involve a larger number of cases and experts from different centers which falls outside the scope of this paper. The goal was simply to demonstrate how the new sequences are perceived by trained eyes. To our knowledge, this is the first time where a similar test can be performed without expecting the obvious identification of the simulated data (cf. with Fig. 1).

\section{B. Image properties: quantitative assessment}

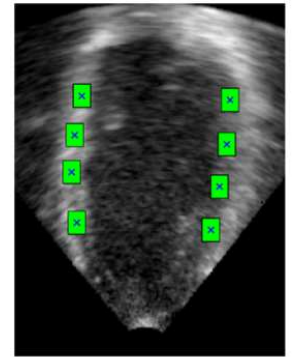

(a)

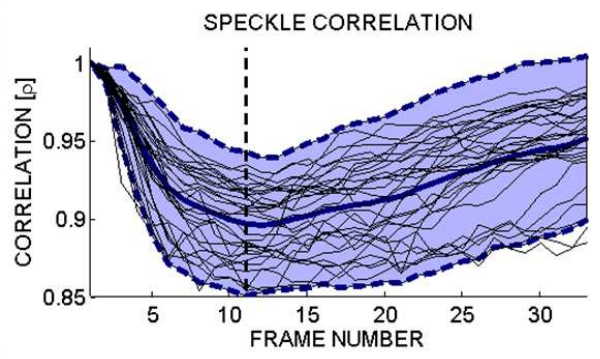

(b)
Fig. 10. (a) Eight 3D patches used to evaluate speckle decorrelation. (b) Time evolution of the correlation coefficient: in black the measurements within each block, in blue the confidence interval $\mu \pm \sigma$. The dashed vertical line denotes end of systole.

1) Speckle statistics: The level of realism was assessed quantitatively by measuring the values of image features commonly exploited for the analysis of cardiac ultrasound datasets. Apart from pixel intensity, first order speckle statistics represent a reliable feature in common tasks as segmentation [47], [48] and motion estimation [49]-[52]. In particular, while blood returns fully developed speckle, leading to the popular Rayleigh model [53], [54], myocardial tissue generates instead partially developed speckle, leading to sub-Rayleigh distributions [43], [47], where the main factor influencing the type of statistical distribution is the number of scattering sites per resolution cell of the ultrasound system [53], [54]. Hereto the local concentration of scatterers was optimized in order to obtain a realistic statistical description of the simulated speckle patterns.

Experimentally we verified that a spatially uniform and sufficiently high concentration of scatterers (i.e. $>4$ scatterers per resolution cel 5 ) was sufficient to generate realistic speckle statistics. Indeed, since scattering amplitude is directly sampled from a speckle image (i.e. the template frame), the desired statistical properties are directly inherited from the latter provided the sampling is dense enough. The local histograms for two image patches extracted from the blood pool and the myocardium are reported in Fig. 8, Each analyzed ROI was taken equal to three times the size of the resolution cell in each direction and comprised $\sim 6800$ samples. Note that histograms were computed on data before subsampling and scan conversion (hence the high number of samples).

\footnotetext{
${ }^{5}$ Resolution cell was taken equal to the extent of the system PSF, whose size in axial $(A)$, azimuth $(W)$ and elevation $(H)$ were computed as: $A=\lambda$, $W=1.02 \lambda F / L$ and $H=W$, being $\lambda$ the wavelength of the transmitted pulse, $F$ the focal distance and $L$ the aperture size [55]. In the performed simulations: $A=0.46 \mathrm{~mm}, W, H=1.9 \mathrm{~mm}$.
}

The analysis showed that computed histograms are in good agreement with the best-fitting distributions known from the literature.

2) Speckle decorrelation: Speckle patterns decorrelate over time due to tissue motion and deformation. This is mainly due to the changes in the relative distance between the scattering centers (i.e. the strain) and, in particular in the case of sectorial probes of use in echocardiography, to the spatial variations of the system PSF [56]. Motion decorrelation weakens the fundamental assumption of many speckle tracking solutions and might negatively impact their performance. Hereto, the level of motion decorrelation present in the proposed sequences was measured.

Note that motion decorrelation is naturally ensured by the proposed framework thanks to the decoupling of the mechanical simulation, establishing the motion of the individual scatterers, from the ultrasound simulation. This is a further advantage from the work in [20] where, by applying the deformation directly to the intensity images, speckle correlation was unrealistically enforced.

We evaluated motion decorrelation by considering eight 3D blocks distributed uniformly along the LV. Blocks were centered on an apical four chamber slice of the simulated 3D volume and their size was $8 \times 4 \times 4 \mathrm{~mm}^{3}(14 \times 6 \times 8$ voxels $)$. The pixels inside each block were then propagated independently over time by using the reference displacement field (i.e. the real tissue motion from the simulated E/M model). The correlation coefficient $\rho$ between the deformed block and the initial one was then measured. The experiment was repeated for the healthy and the ischemic sequences. The obtained correlation curves are reported along with the confidence interval in Fig. 10. As expected in a real setting, correlation decreases until end of systole, when both the change in the distance between the scatterers (as due to strain) and the displacement w.r.t. the original position (hence, the PSF variation) are the largest. These results are qualitatively in agreement with previous simulation studies [56]. Consider that [20] would give by construction correlation values constantly equal to one (absent interpolation artifacts).

\section{Mechanical properties}

The sequence of volumetric meshes, as obtained from the mechanical simulation, defines the ground truth. The reference displacement/strain was computed from a set of seed points sampling the LV myocardium regularly in the radial (R) longitudinal (L) circumferential (C) directions (cf. Fig. 11). The points were displaced according to the ground truth E/M meshes by using baricentric coordinates. Pointwise R, L and $\mathrm{C}$ strains $\left(\epsilon_{R}, \epsilon_{L}\right.$ and $\epsilon_{C}$ respectively) were then measured by the relative change in distance between neighboring points. Namely, $\epsilon_{n}(k)=d_{n}^{k} / d_{n}^{0}-1$ with $d_{n}^{k}$ the distance between two consecutive points along direction $n \in\{R, L, C\}$ at time $k$. No drift correction was applied. Global longitudinal strain (GLS) was computed by measuring length changes of full curves going from base to apex at the endocardium; global circumferential strain (GCS) was measured by the length change of circular curves and global radial strain (GRS) by 


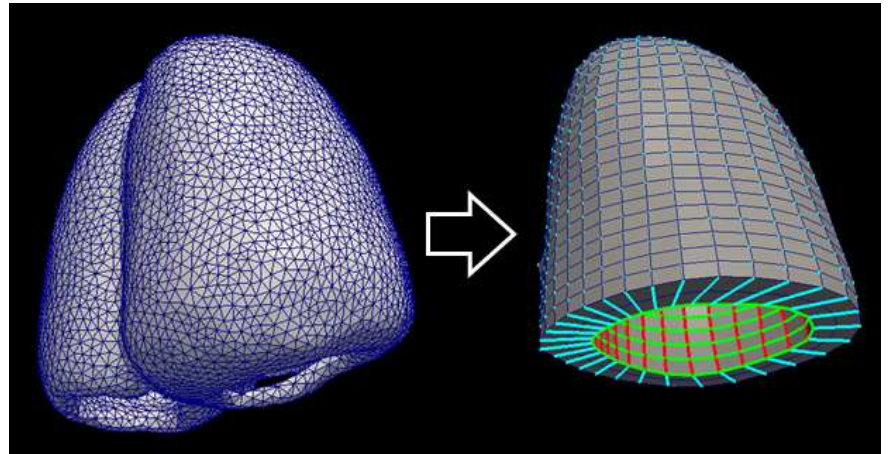

(a) Tetrahedral mesh used in the $E / M$ model

(b) Structured mesh used for strain computation

Fig. 11. Re-meshing of the tetrahedral mesh used for strain computation. Global longitudinal (GLS), circumferential (GCS) and radial (GRS) strains are computed from red, green and cyan lines in (b) respectively.

the length change of segments going from endo- to epicardium (cf. Fig. 11 b)). Segmental values were obtained by cropping these strings by AHA segments [37]. Point strain values at ES were used to create bullseye diagrams.

1) Healthy and ischemic simulations: Regional and global strain curves for the healthy and LADprox ischemic simulations are reported in Fig. 12 and Fig. 13 respectively. The segment color notation in 3(e) is used. The crosses in Fig. 13 denote segments which were set as ischemic in the E/M simulation. As expected in a healthy heart, regional strain curves are homogeneous throughout the muscle, with longitudinal deformations increasing from base to apex [57], [58]. Obtained peak systolic L-strain (Fig. 12, last column) is slightly below the lower limit of normality of $\sim-15 \%$ commonly reported in literature [58]. Comparing against normality range for $\mathrm{C}$ - and $\mathrm{R}$-strain becomes instead extremely difficult given the large variability of the published values. These points are discussed further in the conclusions. Strain curves on the synthetic ischemic case show that the alteration of the electromechanical parameters effectively reduces regional function in the desired territories. This is also evident from the bullseye plots of Fig. 14

2) Dyssynchronous dataset: Segmental L-strain curves for the synchronous and dyssynchronous DLV sequences are reported in Fig. 15] The delayed electrical activation of the lateral wall is well reflected by a timing difference in the strain curves.

\section{Discussions AND CONCLUSION}

We introduced a novel platform for the in silico evaluation and comparison of 3D speckle tracking solutions based on ultra-realistic echocardiographic simulations. The proposed pipeline combines state-of-the art solutions in the fields of electromechanical modeling and ultrasound simulation. The electromechanical model determines the reference values of displacement and strain. The ultrasound simulator in conjunction with a novel technique to compute scattering amplitude ensures realistic speckle patterns and the presence of typical ultrasound artifacts that make motion tracking challenging.

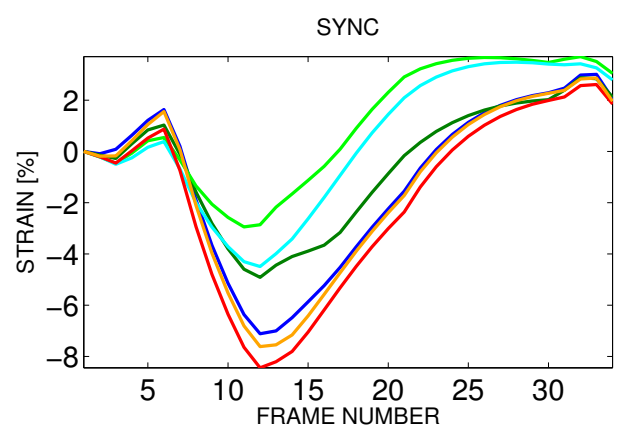

(a) sync

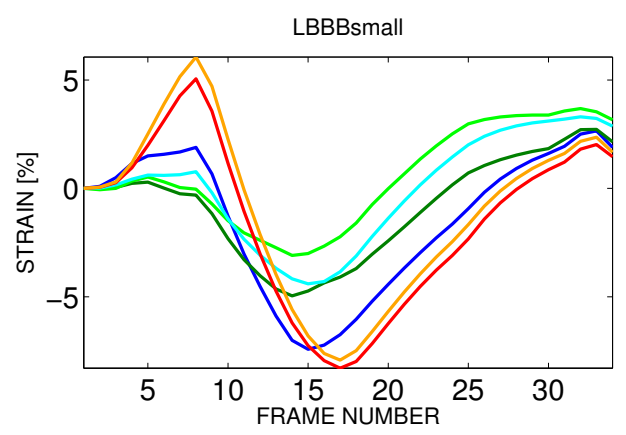

(b) LBBBsmall

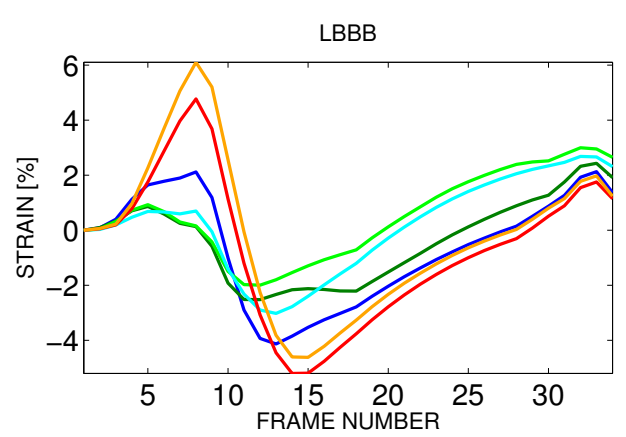

(c) $\mathrm{LBBB}$

Fig. 15. Segmental L-strain curves at mid-wall for the dilated synchronous (a), LBBBsmall (b) and LBBB sequences (c). The delayed activation of the LV lateral (orange and red segments) wall w.r.t. septum (green segments) produces a time shift in the associated strain curves. Segment color notation follows Fig. 3]e)

The pipeline was used to generate an open access database of 8 synthetic sequences, which is available through our project web site: https://team.inria.fr/asclepios/data/straus/

The presented library of sequences contains emblematic cases for two common heart diseases for which 3D speckle tracking has been shown to be a feasible diagnostic tool, namely ischemia and dyssynchrony. Starting from this initial library, our goal is to progressively populate the database with new simulations in order to cover a more exhaustive and diverse range of physiologic and pathological conditions. Also, by progressively modifying the parameters of the electromechanical model and the ultrasound simulator, the sensitivity of 3D strain algorithms to pathology and image properties will be evaluated. Finally, the suitability of the proposed framework to benchmark additional mechanical indexes as transmural strain, LV-twist and torsion as well as less conventional parameters 

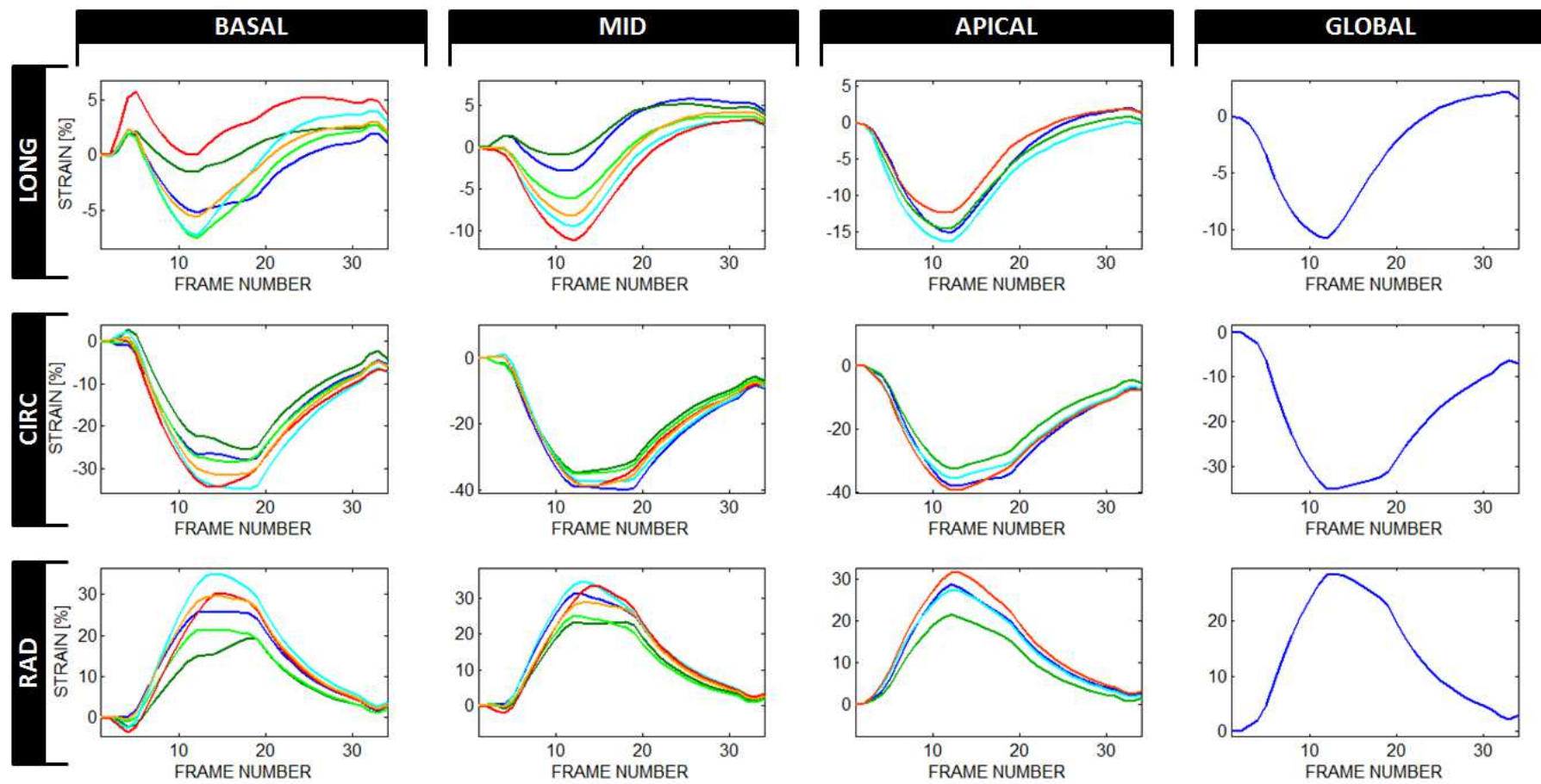

Fig. 12. Regional strain curves for the healthy simulation. Rows denote longitudinal, circumferential and radial directions. Columns refer to basal, mid, top segments and global strain curves respectively. Color notation refers to Fig. 3 e). As expected in a healthy heart, regional strain curves are homogeneous throughout the muscle, with longitudinal deformations increasing from base to apex. Peak systolic L-strain is slightly below the reported ranges of normality while C- and R-strain are slightly above and below the reported ranges (cf. text).
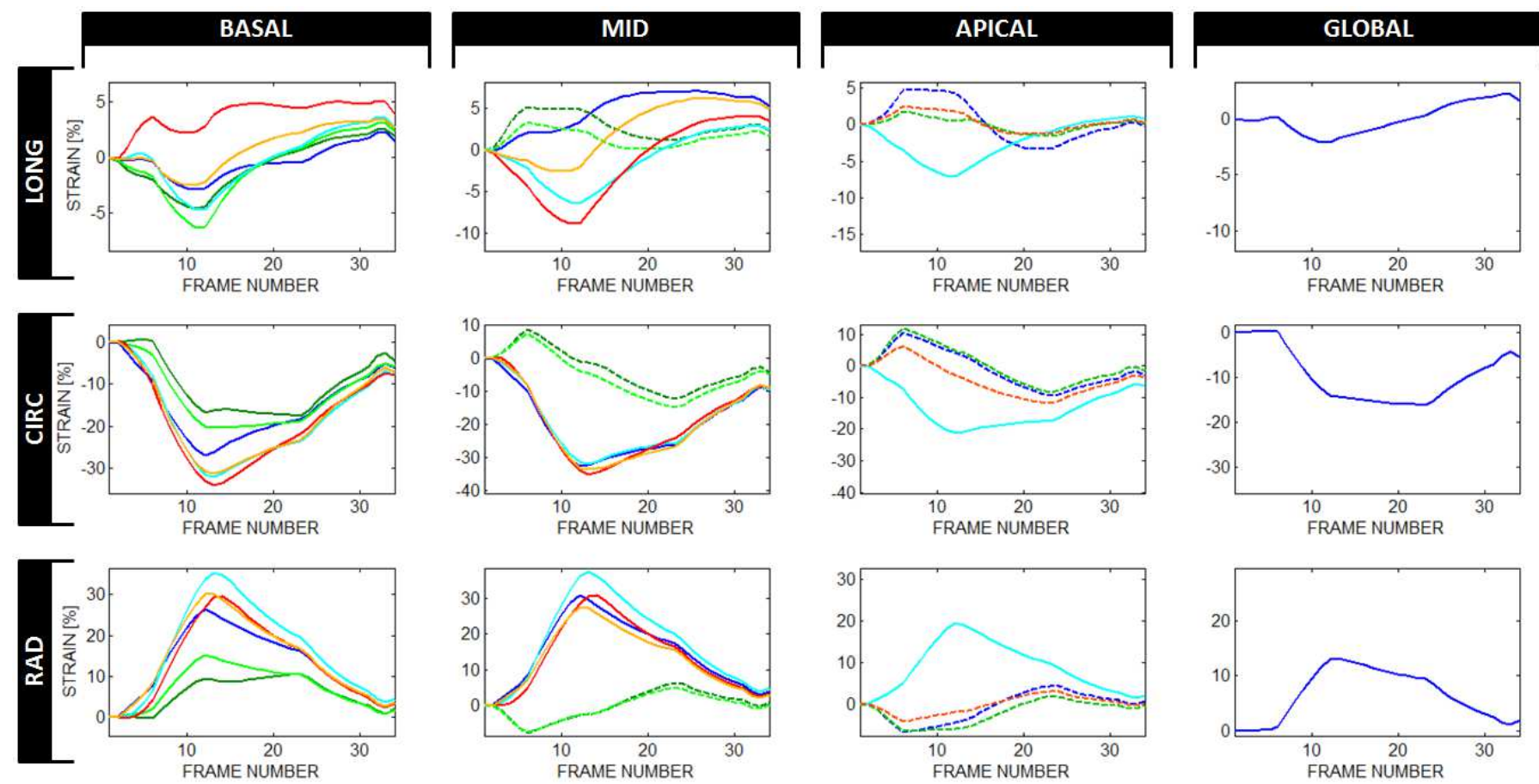

Fig. 13. Regional strain curves for the LADprox simulation. Rows denote longitudinal, circumferential and radial directions. Columns refer to basal, mid, top segments and global strain curves respectively. Color notation refers to Fig. 3 e). Dashed lines denote ischemic segments as established by the E/M simulation (cf. Fig. 3 d)). Strain curves show that the alteration of the electromechanical parameters effectively reduces regional function in the desired territories. Note that simulated ischemia also reduces peak strain values of remote (i.e. non-ischemic) segments and global strain (cf. Fig. 12). 


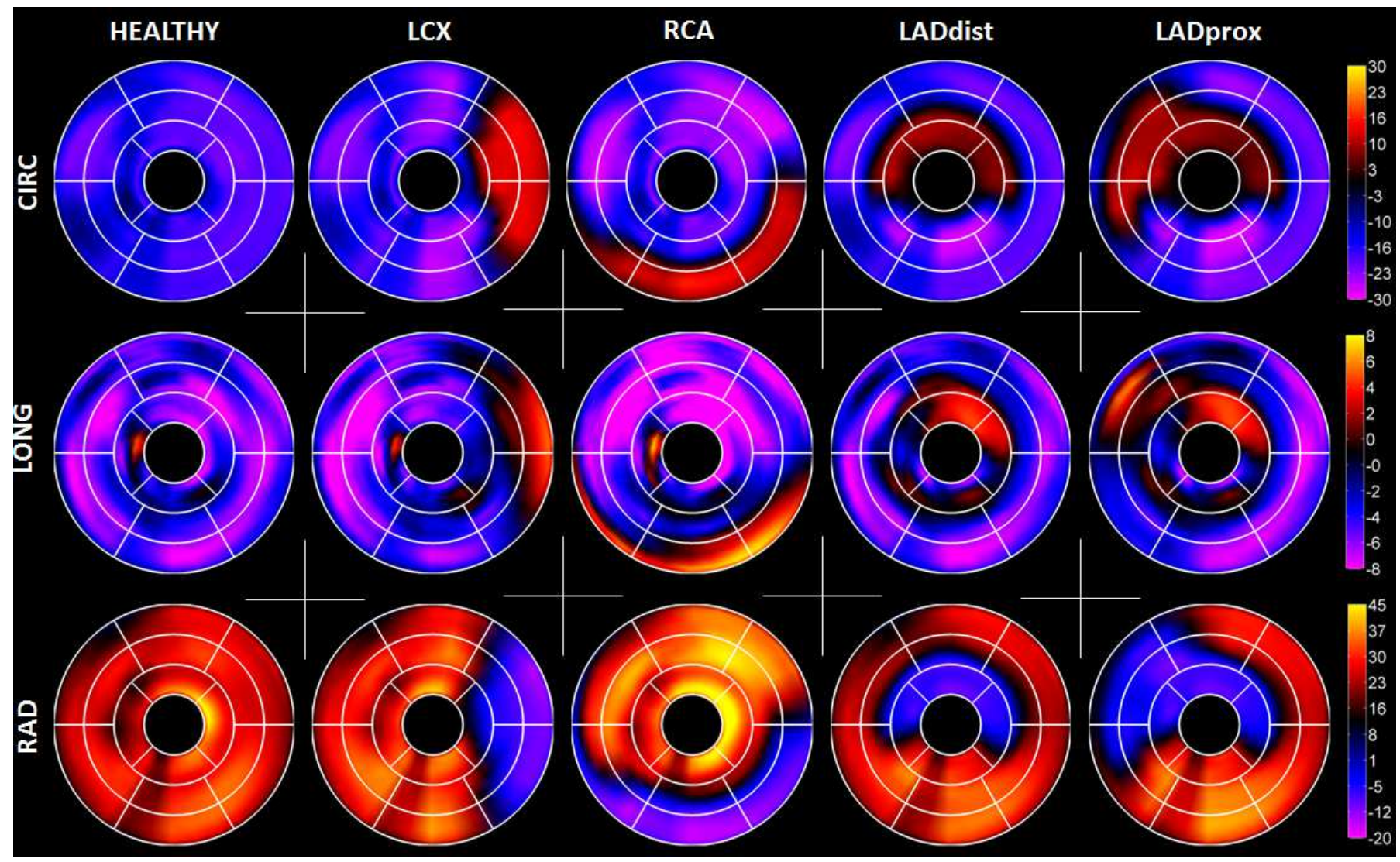

Fig. 14. Bullseye plots of end systolic strain values for healthy and severely ischemic cases. The bullseyes show that the alteration of the electromechanical parameters effectively reduces regional function in the desired territories, cf. Fig. 3

\section{[2] will be investigated.}

Unlike existing databases [19], [21], [30], for each synthetic dataset we will provide B-mode voxel data, raw RF signals and the scatter map used by the ultrasound simulator. RF-tracking has been proven potentially more accurate than B-mode tracking, in particular for the estimation of small displacements [59]-[62]. Nevertheless a thorough and fully reproducible comparison of these techniques is to date still missing. By providing synthetic RF data such a comparison becomes possible. Moreover, COLE [23] was chosen to be a good compromise between accuracy and computational complexity [45]. Nevertheless, COLE exploits several simplifications to the wave propagation equations (linear propagation is assumed, as in FieldII) and in the design of the acquisition scheme. In particular, only traditional single transmit beam-forming can be implemented. As new 3D beam-forming techniques become available, as to increase temporal resolution [63], [64] or to improve speckle trackability [65], [66], testing their impact in the assessment of cardiac function becomes of primary clinical interest. In this perspective more elaborated simulation environment are required [67]-[71]. By making scatter maps available, such alternative simulators can be employed instead of COLE so that most recent advances in terms of ultrasound technology can be readily taken into account. Of note, the increased computational burden associated to these simulators can be mitigated by exploiting existing dedicated online platforms as [72].
The proposed pipeline is completely independent of the ultrasound simulation environment and E/M model used. As such, it can be readily upgraded with the most recent solutions in the two fields. Moreover, although specialized for LV and 3D ultrasound simulations, the proposed pipeline is extremely generic. As such, with only minor modifications, it could be extended to other organs and imaging techniques, provided that adequate motion models, tracking tools and simulation environment exists.

The developed pipeline constitutes a relevant step forward over the state-of-the-art in the field of cardiac ultrasound simulation. Nevertheless its current implementation still presents certain limitations that will be addressed in future studies.

Concerning the electro-mechanical simulation, while global LV function is in good agreement with what was measured experimentally on real datasets (cf. Fig. 5), deformation/strain values remain sub-optimal. In particular, although improvements were made as compared to our previous work [19], peak systolic L-strain still remains low as compared to reported normality ranges [57], [58]. Peak L-strain is to date the most relevant deformation index in clinical diagnostics [73] and hence the optimization of its value will receive privileged attention. Preliminary tests, not reported here, suggest the amount of L-deformation of the E/M model to be strictly correlated with the transmural fiber distribution. Hereto, further effort in personalizing the fiber distribution will be made to improve the realism of the mechanical simulations [74], [75]. 
Circumferential and radial deformations appear to be respectively slightly above and below the normality ranges published in recent studies [57], [58]. Nonetheless, such ranges must be taken with extreme caution given the large variability of the reported values (e.g. an average normal R-strain of $18 \%$ is reported in [76] and of $88 \%$ in [57]). This is mainly due to the absence of well defined guidelines for the assessment of radial and circumferential deformations, especially in 3D. Reaching consensus on definitions and methods for the computation of cardiac deformation is part of the goal this project is aiming to achieve.

A blind test involving four expert readers proved the synthetic sequences to be often indistinguishable from real recordings (cf. Fig. 9). When still screenshots were shown the identification was hard even for trained cardiologists. When the cine-loop was played, the difference became more apparent (yet, with 2 misclassified cases) only for trained cardiologists. Principal sources of identifications were the unrealistically high image quality and the sharp delineation of the endocardial contour. The first point requires further efforts towards the incorporation of typical ultrasound artifacts as clutter noise, dropouts and reverberations. The second point is a consequence of the abrupt change in speckle properties between myocardium (perfectly coherent speckle) and surroundings (completely uncoherent speckle). Hereto, a modified pipeline is under testing where, instead of having a binary transition, a smooth mask setting the ratio between coherent and incoherent speckle components is used.

Tackling these points is also important in the context of benchmarking 3D speckle tracking solutions. Indeed, robustness to artifacts is a key requirement for effective techniques. Moreover, the abrupt transition in speckle properties, on one side, may make the tracking problem harder close to endo- and epicardium while, on the other, it may hinder the automatic detection of the myocardial contours, and hence the overall tracking accuracy, for those tracking techniques making use of a segmentation step [77]. Assuming fully coherent speckle inside the myocardium also neglects the temporal intensity variation due to the changing angle between the cardiac fibers and the acoustic beam. In this sense, a solution would be to express the scattering amplitude as a function of such angle, which is readily computed from the fiber orientation given by the biophysical model.

Ischemic simulations were launched on the healthy geometry. This is correct in the case of early stage ischemia, i.e. before the initiation of remodeling processes that typically follow ischemia and affect heart size and shape [78]. If simulations of acute infarction or recurrent ischemia are carried out instead, the adopted template geometry should be better chosen from a representative class of patients.

A consequence of using a real recording as a template is the biasing of the image quality towards the one of the particular equipment used in the acquisition (a Philips iE33 ultrasound machine with a X5 matrix array transducer in our case). Instead of being a limitation, this feature could open the way to new synthetic inter-vendor studies. Inter-vendor variability is indeed one of the main factors still holding back adoption of 3D speckle tracking in the clinical practice [1].
Besides serving as a benchmarking tool for motion estimators, the same pipeline presented here would be relevant in different kinds of studies. At first, it provides an easy way to generate an arbitrarily large training databases for model based segmentation and tracking algorithms [79]. Moreover, since the E/M simulations embed a known electrical activation sequence, the proposed framework can be used to benchmark algorithms for ultrasound-based cardiac activation mapping [80], [81]. Finally, integrated with adequate navigation functions, our pipeline could be exploited to build software tools for training physicians in the use of $3 \mathrm{D}$ cardiac ultrasound [82].

Immediate future work include the direct comparison of state-of-the-art strain estimation techniques with the new pipeline, on the line of [19]. In particular, of interest is assessing a clear understanding of benefits and limitations of techniques based on block matching, which represents the standard implementation on commercial systems, versus techniques based on elastic registration. Elastic registration has shown potential advantages over block matching but its employment is still confined to the research scenario. A fully reliable and reproducible comparison of these two families of techniques is to date still missing in literature. Initial results of this comparison study have been reported in [83].

An extension of the proposed framework to the simulation of $2 \mathrm{D}$ ultrasound recordings is also being developed. Besides providing a reliable benchmark to $2 \mathrm{D}$ speckle tracking algorithms, the 2D sequences could be used to better evaluate the pitfalls of the $2 \mathrm{D}$ modality with respect to $3 \mathrm{D}$ for the assessment of cardiac function. Comparing B-mode and RF tracking solutions will be also object of future studies.

\section{ACKNOWLEDGMENT}

The work of Martino Alessandrini was supported by the Fonds Wetenschappelijk Onderzoek (1263814N). The research leading to these results has received funding from the EU FP7 for research, technological development and demonstration under grant agreement VP2HF (no 611823) and MedYMA (ERC 2011-291080). The authors would like to thank Francesca Galluzzo, Ph.D.; Adrian Basarab, Ph.D.; Frédéric Schnell, MD; and Efstathios Pagkourelias, MD, for participating in the blind test.

\section{APPENDIX A}

\section{ELECTROMECHANICAL MODEL PARAMETERS AND OPEN} ACCESS DATABASE

The values of the mechanical parameters used in the E/M simulations are summarized in Table II More details on their physiological meaning can be found in [22].

\section{APPENDIX B}

\section{DETAILED RESULTS OF THE BLIND TEST}

The detailed results of the bind test for still screenshots and cine-loops are reported in Table III and Table IV respectively. True positives (TP, i.e. correctly identified simulations), true negatives (TN), false positives (FP) and false negatives (FN) are reported for each observer along with the corresponding 
TABLE II

MECHANICAL PARAMETERS EMPLOYED FOR HEALTHY (NORM.) MILDLY ISCHEMIC (ISCH) AND SEVERELY ISCHEMIC (ISCH+) SEGMENTS IN THE NORMAL GEOMETRY AND FOR THE DILATED GEOMETRY (DLV). SEE [22] FOR MORE DETAILS.

\begin{tabular}{cll|l|l|l|l} 
Symbol & Description & Units & Norm. & Isch & Isch+ & DLV \\
\hline$\sigma_{0}$ & $\begin{array}{l}\text { maximum } \\
\text { contraction }\end{array}$ & $\mathrm{Pa}$ & $3.7 \mathrm{e} 6$ & $1 \mathrm{e} 5$ & $5 \mathrm{e} 4$ & $2.5 \mathrm{e} 6$ \\
$\kappa_{0}$ & $\begin{array}{l}\text { maximum } \\
\text { stiffness } \\
\kappa_{\text {atp }}\end{array}$ & $\mathrm{Pa}$ & $6 \mathrm{e} 6$ & $1 \mathrm{e} 6$ & $1 \mathrm{e} 5$ & $6 \mathrm{e} 6$ \\
$\kappa_{r s}$ & $\begin{array}{l}\text { ratraction } \\
\text { relaxation } \\
\text { rate }\end{array}$ & $\mathrm{s}^{-1}$ & 30 & 20 & 20 & 30 \\
\hline
\end{tabular}

values of sensitivity (SE), specificity (SP) and accuracy (ACC). Expert observers are differentiated by clinical (C) and technical (T) background.

TABLE III

BLIND TEST RESULTS FOR STILL FRAMES.

\begin{tabular}{|c|c|c|c|c|c|c|c|}
\hline Observer & $\mathrm{TP}$ & $\mathrm{TN}$ & FP & FN & SP & SE & $\mathrm{ACC}$ \\
\hline $\mathrm{C} 1$ & 3 & 6 & 1 & 4 & $86 \%$ & $43 \%$ & $64 \%$ \\
\hline $\mathrm{C} 2$ & 3 & 2 & 5 & 4 & $29 \%$ & $43 \%$ & $36 \%$ \\
\hline $\mathrm{T} 1$ & 3 & 0 & 7 & 4 & $0 \%$ & $43 \%$ & $21 \%$ \\
\hline $\mathrm{T} 2$ & 3 & 5 & 2 & 4 & $71 \%$ & $43 \%$ & $57 \%$ \\
\hline
\end{tabular}

TABLE IV

BLIND TEST RESULTS FOR CINE LOOPS. THE ONLY ANSWER OF TYPE "UNKNOWN" WAS RECEIVED FROM T2 AND WAS NOT INCLUDED IN THE ANALYSIS

\begin{tabular}{|c|c|c|c|c|c|c|c|}
\hline Observer & $\mathrm{TP}$ & $\mathrm{TN}$ & FP & $\mathrm{FN}$ & SP & SE & ACC \\
\hline $\mathrm{C} 1$ & 4 & 4 & 0 & 0 & $100 \%$ & $100 \%$ & $100 \%$ \\
\hline $\mathrm{C} 2$ & 4 & 2 & 2 & 0 & $50 \%$ & $100 \%$ & $75 \%$ \\
\hline $\mathrm{T} 1$ & 0 & 2 & 2 & 4 & $50 \%$ & $0 \%$ & $25 \%$ \\
\hline $\mathrm{T} 2$ & 2 & 2 & 1 & 2 & $66 \%$ & $50 \%$ & $57 \%$ \\
\hline
\end{tabular}

\section{REFERENCES}

[1] V. Mor-Avi, R. M. Lang, L. P. Badano, M. Belohlavek, N. M. Cardim, G. Derumeaux, M. Galderisi, T. Marwick, S. F. Nagueh, P. P. Sengupta, R. Sicari, O. A. Smiseth, B. Smulevitz, M. Takeuchi, J. D. Thomas, M. Vannan, J.-U. Voigt, and J. L. Zamorano, "Current and evolving echocardiographic techniques for the quantitative evaluation of cardiac mechanics: ASE/EAE consensus statement on methodology and indications: Endorsed by the japanese society of echocardiography," Journal of the American Society of Echocardiography, vol. 24, no. 3, pp. 277 $313,2011$.

[2] R. Jasaityte, P. Claus, A. J. Teske, L. Herbots, B. Verheyden, R. Jurcut, F. Rademakers, and J. D'hooge, "The slope of the segmental stretchstrain relationship as a noninvasive index of $\{\mathrm{LV}\}$ inotropy," JACC: Cardiovascular Imaging, vol. 6, no. 4, pp. 419 - 428, 2013.

[3] G. R. Sutherland, G. Di Salvo, P. Claus, J. D'hooge, and B. Bijnens, "Strain and strain rate imaging: a new clinical approach to quantifying regional myocardial function," J Am Soc Echocardiogr, vol. 17, no. 7, pp. 788-802, 2004.

[4] P. Ballo, A. Motto, S. Mondillo, and M. Galderisi, "Three-dimensional echocardiography for optimization of cardiac resynchronization therapy," European Heart Journal, vol. 30, no. 4, p. 505, 2009.

[5] E. Gayat, H. Ahmad, L. Weinert, R. M. Lang, and V. Mor-Avi, "Reproducibility and inter-vendor variability of left ventricular deformation measurements by three-dimensional speckle-tracking echocardiography," J Am Soc Echocardiogr, vol. 24, no. 8, pp. 878 - 885, 2011.
[6] L. Herbots, F. Maes, J. D'hooge, P. Claus, S. Dymarkowski, P. Mertens, L. Mortelmans, B. Bijnens, J. Bogaert, F. Rademakers, and G. Sutherland, "Quantifying myocardial deformation throughout the cardiac cycle: a comparison of ultrasound strain rate, grey-scale m-mode and magnetic resonance imaging," Ultrasound in Medicine and Biology, vol. 30, no. 5, pp. 591-598, 2004.

[7] B. H. Amundsen, T. Helle-Valle, T. Edvardsen, H. Torp, J. Crosby, E. Lyseggen, A. Stoylen, H. Ihlen, J. Lima, O. A. Smiseth, and S. A. Slordahl, "Noninvasive myocardial strain measurement by speckle tracking echocardiography: Validation against sonomicrometry and tagged magnetic resonance imaging," Journal of the American College of Cardiology, vol. 47, no. 4, pp. $789-793,2006$.

[8] S. Langeland, J. Dhooge, P. F. Wouters, H. A. Leather, P. Claus, B. Bijnens, and G. R. Sutherland, "Experimental validation of a new ultrasound method for the simultaneous assessment of radial and longitudinal myocardial deformation independent of insonation angle," Circulation, vol. 112, pp. 2157-2162, 2005.

[9] Q. Duan, K. Parker, A. Lorsakul, E. Angelini, E. Hyodo, S. Homma, J. Holmes, and A. Laine, "Quantitative validation of optical flow based myocardial strain measures using sonomicrometry," in Biomedical Imaging: From Nano to Macro, 2009. ISBI '09. IEEE International Symposium on, 2009, pp. 454-457.

[10] B. Heyde, R. Jasaityte, D. Barbosa, V. Robesyn, S. Bouchez, P. Wouters, F. Maes, P. Claus, and J. D'hooge, "Elastic image registration versus speckle tracking for 2-d myocardial motion estimation: A direct comparison in vivo," Medical Imaging, IEEE Transactions on, vol. 32, no. 2, pp. 449-459, 2013.

[11] B. Lesniak Plewinska, S. Cygan, K. Kaluzynski, J. D’hooge, J. Zmigrodzki, E. Kowalik, M. Kordybach, and M. Kowalski, "A dual-chamber, thick-walled cardiac phantom for use in cardiac motion and deformation imaging by ultrasound," Ultrasound Med Biol, vol. 36, no. 7, pp. 11451156, 2010.

[12] B. Heyde, S. Cygan, H. F. Choi, B. Lesniak-Plewinska, D. Barbosa, A. Elen, P. Claus, D. Loeckx, K. Kaluzynski, and J. D'hooge, "Regional cardiac motion and strain estimation in three-dimensional echocardiography: a validation study in thick-walled univentricular phantoms," Ultrasonics, Ferroelectrics and Frequency Control, IEEE Transactions on, vol. 59, no. 4, pp. 668-682, 2012.

[13] J. de Hart, A. de Weger, S. van Tuijl, J. Stijnen, C. van den Broek, M. Rutten, and B. de Mol, "An ex vivo platform to simulate cardiac physiology: a new dimension for therapy development and assessment," Int J Artif Organs, vol. 34, no. 6, pp. 495 - 505, 2011.

[14] A. Leopaldi, R. Vismara, M. Lemma, L. Valerio, M. Cervo, A. Mangini, M. Contino, A. Redaelli, C. Antona, and G. Fiore, "In vitro hemodynamics and valve imaging in passive beating hearts," Journal of Biomechanics, vol. 45, no. 7, pp. 1133 - 1139, 2012.

[15] A. Richards, R. Cook, G. Bolotin, and G. Buckner, "A dynamic heart system to facilitate the development of mitral valve repair techniques," Annals of Biomedical Engineering, vol. 37, no. 4, pp. 651-660, 2009.

[16] A. Elen, H. F. Choi, D. Loeckx, H. Gao, P. Claus, P. Suetens, F. Maes, and J. D'hooge, "Three-dimensional cardiac strain estimation using spatio temporal elastic registration of ultrasound images: A feasibility study," Medical Imaging, IEEE Transactions on, vol. 27, no. 11, pp. 1580-1591, 2008.

[17] Q. Duan, P. Moireau, E. Angelini, D. Chapelle, and A. Laine, "Simulation of 3D ultrasound with a realistic electro-mechanical model of the heart," in Functional Imaging and Modeling of the Heart, ser. Lecture Notes in Computer Science, F. Sachse and G. Seemann, Eds. Springer Berlin Heidelberg, 2007, vol. 4466, pp. 463-473.

[18] M. Alessandrini, A. Basarab, H. Liebgott, and O. Bernard, "Myocardial motion estimation from medical images using the monogenic signal," Image Processing, IEEE Transactions on, vol. 22, no. 3, pp. 1084-1095, 2013.

[19] M. De Craene, S. Marchesseau, B. Heyde, H. Gao, M. Alessandrini, O. Bernard, G. Piella, A. Porras, L. Tautz, A. Hennemuth, A. Prakosa, H. Liebgott, O. Somphone, P. Allain, S. Makram Ebeid, H. Delingette, M. Sermesant, J. D'hooge, and E. Saloux, "3D strain assessment in ultrasound (straus): A synthetic comparison of five tracking methodologies," Medical Imaging, IEEE Transactions on, vol. 32, no. 9, pp. 1632-1646, 2013.

[20] A. Prakosa, M. Sermesant, H. Delingette, S. Marchesseau, E. Saloux, P. Allain, N. Villain, and N. Ayache, "Generation of synthetic but visually realistic time series of cardiac images combining a biophysical model and clinical images," Medical Imaging, IEEE Transactions on, vol. 32, no. 1, pp. 99-109, 2013.

[21] M. Alessandrini, H. Liebgott, D. Friboulet, and O. Bernard, "Simulation of realistic echocardiographic sequences for ground-truth validation 
of motion estimation," in Image Processing (ICIP), 2012 19th IEEE International Conference on, Sept 2012, pp. 2329-2332.

[22] S. Marchesseau, H. Delingette, M. Sermesant, M. Sorine, K. Rhode, S. Duckett, C. Rinaldi, R. Razavi, and N. Ayache, "Preliminary specificity study of the Bestel-Clement-Sorine electromechanical model of the heart using parameter calibration from medical images," J Mech Behav Biomed Mater, vol. 20, pp. 259-271, 2013.

[23] H. Gao, H. F. Choi, P. Claus, S. Boonen, S. Jaecques, G. Van Lenthe, G. Van der Perre, W. Lauriks, and J. D'hooge, "A fast convolutionbased methodology to simulate 2D/3D cardiac ultrasound images," Ultrasonics, Ferroelectrics and Frequency Control, IEEE Transactions on, vol. 56, no. 2, pp. 404-409, 2009.

[24] P. Baraldi, A. Sarti, C. Lamberti, A. Prandini, and F. Sgallari, "Evaluation of differential optical flow techniques on synthesized echo images," Biomedical Engineering, IEEE Transactions on, vol. 43, no. 3, pp. 259272, 1996.

[25] M. Suhling, M. Arigovindan, C. Jansen, P. Hunziker, and M. Unser, "Myocardial motion analysis from B-mode echocardiograms," Image Processing, IEEE Transactions on, vol. 14, no. 4, pp. 525-536, 2005.

[26] J. Sainte-Marie, D. Chapelle, R. Cimrman, and M. Sorine, "Modeling and estimation of the cardiac electromechanical activity," Computers \& Structures, vol. 84, no. 28, pp. 1743 - 1759, 2006.

[27] J. Jensen and N. Svendsen, "Calculation of pressure fields from arbitrarily shaped, apodized, and excited ultrasound transducers," Ultrasonics, Ferroelectrics and Frequency Control, IEEE Transactions on, vol. 39, no. 2, pp. 262-267, 1992.

[28] J. A. Jensen, "Field: A program for simulating ultrasound systems," in 10th nordicbaldic conference on biomedical imaging, vol. 4, supplement 1, part 1:351-353, 1996, pp. 351-353.

[29] M. Ledesma-Carbayo, J. Kybic, M. Desco, A. Santos, M. Suhling, P. Hunziker, and M. Unser, "Spatio-temporal nonrigid registration for ultrasound cardiac motion estimation," Medical Imaging, IEEE Transactions on, vol. 24, no. 9, pp. 1113-1126, Sept 2005.

[30] M. De Craene, M. Alessandrini, P. Allain, S. Marchesseau, I. WaechterStehle, J. Weese, E. Saloux, H. G. Morales, R. Cuingnet, H. Delingette, M. Sermesant, O. Bernard, and J. D'hooge, "Ultra-realistic synthetic echocardiographic sequences for quality assurance of strain estimation algorithms," in Biomedical Imaging: From Nano to Macro, 2009. ISBI '14. IEEE International Symposium on, 2014, pp. 73-76.

[31] O. Ecabert, J. Peters, H. Schramm, C. Lorenz, J. Von Berg, M. Walker, M. Vembar, M. Olszewski, K. Subramanyan, G. Lavi, and J. Weese, "Automatic model-based segmentation of the heart in CT images," IEEE TMI, vol. 27, no. 9, pp. 1189-1201, 2008 .

[32] W. Tsang, I. S. Salgo, L. Zarochev, S. Settlemier, N. Bhave, J. Weese, I. Waechter-Stehle, M. Cardinale, A. Prado, L. Weinert, A. R. Patel, and R. M. Lang, "Fully automated quantification of left ventricular and left atrial volumes from transthoracic 3D echocardiography: A validation study," J Am Coll Cardiol., no. 61, 2013.

[33] F. Weber, T. Stehle, I. Waechter-Stehle, M. Gtz, J. Peters, S. Mollus, J. Balzer, M. Kelm, and J. Weese, "Analysis of mitral valve motion in $4 \mathrm{~d}$ transesophageal echocardiography for transcatheter aortic valve implantation," in Statistical Atlases and Computational Models of the Heart - Imaging and Modelling Challenges, ser. Lecture Notes in Computer Science, O. Camara, T. Mansi, M. Pop, K. Rhode, M. Sermesant, and A. Young, Eds. Springer International Publishing, 2015, pp. 168-176. [Online]. Available: http://dx.doi.org/10.1007/978-3-319-14678-2_17

[34] L. G. Rudski, W. W. Lai, J. Afilalo, L. Hua, M. D. Handschumacher, K. Chandrasekaran, S. D. Solomon, E. K. Louie, and N. B. Schiller, "Guidelines for the echocardiographic assessment of the right heart in adults: A report from the american society of echocardiography: Endorsed by the european association of echocardiography, a registered branch of the european society of cardiology, and the canadian society of echocardiography," Journal of the American Society of Echocardiography, vol. 23, no. 7, pp. $685-713,2010$.

[35] Q. Fang and D. Boas, "Tetrahedral mesh generation from volumetric binary and grayscale images," in Biomedical Imaging: From Nano to Macro, 2009. ISBI '09. IEEE International Symposium on, 2009, pp. $1142-1145$

[36] M. Sermesant, R. Chabiniok, P. Chinchapatnam, T. Mansi, F. Billet, P. Moireau, J. Peyrat, K. Wong, J. Relan, K. Rhode, M. Ginks, P. Lambiase, H. Delingette, M. Sorine, C. Rinaldi, D. Chapelle, R. Razavi, and N. Ayache, "Patient-specific electromechanical models of the heart for the prediction of pacing acute effects in CRT: A preliminary clinical validation," Medical Image Analysis, vol. 16, no. 1, pp. 201 - 215, 2012.

[37] AHA Writing Group on Myocardial Segmentation and Registration for Cardiac Imaging, M. D. Cerqueira, N. J. Weissman, V. Dilsizian,
A. K. Jacobs, S. Kaul, W. K. Laskey, D. J. Pennell, J. A. Rumberger, T. Ryan, and M. S. Verani, "Standardized myocardial segmentation and nomenclature for tomographic imaging of the heart: A statement for healthcare professionals from the cardiac imaging committee of the council on clinical cardiology of the american heart association," Circulation, vol. 105, no. 4, pp. 539-542, 2002.

[38] L. D. Jacobs, I. S. Salgo, S. Goonewardena, L. Weinert, P. Coon, D. Bardo, O. Gerard, P. Allain, J. L. Zamorano, L. P. de Isla, V. Mor-Avi, and R. M. Lang, "Rapid online quantification of left ventricular volume from real-time three-dimensional echocardiographic data," European Heart Journal, vol. 27, no. 4, pp. 460-468, 2006.

[39] O. Somphone, M. De Craene, R. Ardon, B. Mory, P. Allain, H. Gao, J. D'hooge, S. Marchesseau, M. Sermesant, H. Delingette, and E. Saloux, "Fast myocardial motion and strain estimation in 3D cardiac ultrasound with sparse demons," in Biomedical Imaging (ISBI), 2013 IEEE 10th International Symposium on, April 2013, pp. 1182-1185.

[40] F. L. Bookstein, "Principal warps: thin-plate splines and the decomposition of deformations," Pattern Analysis and Machine Intelligence, IEEE Transactions on, vol. 11, no. 6, pp. 567-585, 1989.

[41] J. P. Lewis, F. Pighin, and K. Anjyo, "Scattered data interpolation and approximation for computer graphics," in ACM SIGGRAPH ASIA 2010 Courses, ser. SA '10. New York, NY, USA: ACM, 2010, pp. 2:1-2:73.

[42] H. S. M. Coxeter, Introduction to Geometry, 2nd ed. Wiley Classics Library, 1962.

[43] O. Bernard, B. Touil, J. D'hooge, and D. Friboulet, "Statistical modeling of the radio-frequency signal for partially- and fully-developed speckle based on a generalized gaussian model with application to echocardiography," Ultrasonics, Ferroelectrics and Frequency Control, IEEE Transactions on, vol. 54, no. 10, pp. 2189-2194, 2007.

[44] R. Molthen, V. Manoj Narayanan, P. Shankar, J. Reid, V. Genis, and L. Vergara-Dominguez, "Ultrasound echo evaluation by k-distribution," in Ultrasonics Symposium, 1993. Proceedings., IEEE 1993, 1993, pp. 957-960 vol.2.

[45] H. Gao, T. Hergum, H. Torp, and J. Dhooge, "Comparison of the performance of different tools for fast simulation of ultrasound data," Ultrasonics, vol. 52, no. 5, pp. 573 - 577, 2012.

[46] O. Bernard, B. Heyde, M. Alessandrini, D. Barbosa, S. CamarasuPop, F. Cervenansky, S. Valette, O. Mirea, E. Galli, M. Geleijnse, A. Papachristidis, J. Bosch, and J. D'hooge, "Challenge on endocardial three-dimensional ultrasound segmentation (CETUS)," in Proc. of MICCAI Challenge on Echocardiographic Three-Dimensional Ultrasound Segmentation (CETUS). MIDAS journal, 2014, pp. $1-8$.

[47] O. Bernard, J. D'hooge, and D. Friboulet, "Statistics of the radiofrequency signal based on $\mathrm{K}$ distribution with application to echocardiography," Ultrasonics, Ferroelectrics and Frequency Control, IEEE Transactions on, vol. 53, no. 9, pp. $1689-1694$, sept. 2006.

[48] M. Alessandrini, D. Friboulet, O. Basset, J. D'hooge, and O. Bernard, "Level-set segmentation of myocardium and epicardium in ultrasound images using localized Bhattacharyya distance," in Ultrasonics Symposium (IUS), 2009 IEEE International, 2009, pp. 2468-2471.

[49] M. Strintzis and I. Kokkinidis, "Maximum likelihood motion estimation in ultrasound image sequences," Signal Processing Letters, IEEE, vol. 4, no. 6, pp. 156-157, 1997.

[50] B. Cohen and I. Dinstein, "New maximum likelihood motion estimation schemes for noisy ultrasound images," Pattern Recognition, vol. 35, no. 2 , pp. $455-463,2002$.

[51] A. Myronenko, X. Song, and D. Sahn, "Maximum likelihood motion estimation in 3D echocardiography through non-rigid registration in spherical coordinates," in Functional Imaging and Modeling of the Heart, ser. Lecture Notes in Computer Science, N. Ayache, H. Delingette, and M. Sermesant, Eds. Springer Berlin Heidelberg, 2009, vol. 5528, pp. $427-436$.

[52] G. Piella, A. Porras, M. Craene, N. Duchateau, and A. Frangi, "Temporal diffeomorphic free form deformation to quantify changes induced by left and right bundle branch block and pacing," in Statistical Atlases and Computational Models of the Heart. Imaging and Modelling Challenges, ser. Lecture Notes in Computer Science, O. Camara, T. Mansi, M. Pop, K. Rhode, M. Sermesant, and A. Young, Eds. Springer Berlin Heidelberg, 2013, vol. 7746, pp. 134-141.

[53] J. W. Goodman, Statistical Optics. Wiley Classic Library Edition, 2000.

[54] R. Wagner, S. Smith, J. Sandrik, and H. Lopez, "Statistics of speckle in ultrasound B-scans," Sonics and Ultrasonics, IEEE Transactions on, vol. 30 , no. 3, pp. 156-163, 1983 .

[55] T. L. Szabo, "Chapter 6 - beamforming," in Diagnostic Ultrasound Imaging: Inside Out (Second Edition), second edition ed., T. L. Szabo, Ed. Boston: Academic Press, 2014, pp. 167 - 207. 
[56] B. Touil, A. Basarab, P. Delachartre, O. Bernard, and D. Friboulet, "Analysis of motion tracking in echocardiographic image sequences: Influence of system geometry and point-spread function," Ultrasonics, vol. 50, no. 3, pp. 373 - 386, 2010.

[57] K. Kaku, M. Takeuchi, W. Tsang, K. Takigiku, S. Yasukochi, A. R. Patel, V. Mor-Avi, R. M. Lang, and Y. Otsuji, "Age-related normal range of left ventricular strain and torsion using three-dimensional speckletracking echocardiography," J Am Soc Echocardiogr, vol. 27, pp. 55-64, jan 2014.

[58] D. Muraru, U. Cucchini, S. Mihil, M. H. Miglioranza, P. Aruta, G. Cavalli, A. Cecchetto, S. Padayattil-Jos, D. Peluso, S. Iliceto, and L. P. Badano, "Left ventricular myocardial strain by three-dimensional speckle-tracking echocardiography in healthy subjects: Reference values and analysis of their physiologic and technical determinants," Journal of the American Society of Echocardiography, vol. 27, no. 8, pp. 858 871.e1, 2014.

[59] J. D’hooge, A. Heimdal, F. Jamal, T. Kukulski, B. Bijnens, F. Rademakers, L. Hatle, P. Suetens, and G. R. Sutherland, "Regional Strain and Strain Rate Measurements by Cardiac Ultrasound: Principles, Implementation and Limitations," European Journal of Echocardiography, vol. 1 , no. 3, pp. $154-170$, Sep. 2000.

[60] E. Konofagou and J. Ophir, "A new elastographic method for estimation and imaging of lateral displacements, lateral strains, corrected axial strains and poissons ratios in tissues," Ultrasound in Medicine \& Biology, vol. 24, no. 8, pp. 1183 - 1199, 1998.

[61] R. G. Lopata, M. M. Nillesen, J. M. Thijssen, L. Kapusta, and C. L. de Korte, "Three-dimensional cardiac strain imaging in healthy children using RF-data," Ultrasound in Medicine \& Biology, vol. 37, no. 9, pp. 1399 - 1408, 2011.

[62] B. Heyde, M. Alessandrini, L. Tong, and J. D'hooge, "RF-based motion estimation using non-rigid image registration techniques: Insilico and in-vivo feasibility," in Ultrasonics Symposium (IUS), 2014 IEEE International, Sept 2014, pp. 2280-2283.

[63] C. Papadacci, M. Pernot, M. Couade, M. Fink, and M. Tanter, "Highcontrast ultrafast imaging of the heart," Ultrasonics, Ferroelectrics and Frequency Control, IEEE Transactions on, vol. 61, no. 2, pp. 288-301, February 2014.

[64] L. Tong, A. Ramalli, R. Jasaityte, P. Tortoli, and J. D'hooge, "Multitransmit beam forming for fast cardiac imaging - experimental demonstration and in-vivo application," Medical Imaging, IEEE Transactions on, in press.

[65] H. Liebgott, A. Basarab, P. Gueth, D. Friboulet, and P. Delachartre, "Transverse oscillations for tissue motion estimation," Ultrasonics, vol. 50 , no. 6 , pp. $548-555,2010$

[66] M. Alessandrini, A. Basarab, L. Boussel, X. Guo, A. Serusclat, D. Friboulet, D. Kouame, O. Bernard, and H. Liebgott, "A new technique for the estimation of cardiac motion in echocardiography based on transverse oscillations: A preliminary evaluation in silico and a feasibility demonstration in vivo," Medical Imaging, IEEE Transactions on, vol. 33, no. 5, pp. 1148-1162, May 2014

[67] J. Jensen and N. Svendsen, "Calculation of pressure fields from arbitrarily shaped, apodized, and excited ultrasound transducers," Ultrasonics, Ferroelectrics, and Frequency Control, IEEE Transactions on, vol. 39, no. 2, pp. 262-267, March 1992.

[68] F. Varray, C. Cachard, A. Ramalli, P. Tortoli, and O. Basset, "Simulation of ultrasound nonlinear propagation on gpu using a generalized angular spectrum method," EURASIP J Image Video Process, vol. 2011, no. 17, pp. 1-6, 2011

[69] T. Bruyneel, A. Ortega, L. Tong, and J. D'hooge, "A GPU-based implementation of the spatial impulse response method for fast calculation of linear sound fields and pulse-echo responses of array transducers," in Ultrasonics Symposium (IUS), 2013 IEEE International, July 2013, pp. 367-369.

[70] B. E. Treeby, J. Jaros, A. P. Rendell, and B. T. Cox, "Modeling nonlinear ultrasound propagation in heterogeneous media with power law absorption using a k-space pseudospectral method," The Journal of the Acoustical Society of America, vol. 131, no. 6, pp. 4324-4336, 2012.

[71] T. Hergum, S. Langeland, E. Remme, and H. Torp, "Fast ultrasound imaging simulation in k-space," Ultrasonics, Ferroelectrics, and Frequency Control, IEEE Transactions on, vol. 56, no. 6, pp. 1159-1167, June 2009.

[72] T. Glatard, C. Lartizien, B. Gibaud, R. Ferreira da Silva, G. Forestier, F. Cervenansky, M. Alessandrini, H. Benoit-Cattin, O. Bernard, S. Camarasu-Pop, N. Cerezo, P. Clarysse, A. Gaignard, P. Hugonnard, H. Liebgott, S. Marache, A. Marion, J. Montagnat, J. Tabary, and D. Friboulet, "A virtual imaging platform for multi-modality medical image simulation," Medical Imaging, IEEE Transactions on, vol. 32, no. 1, pp. 110-118, Jan 2013.

[73] J. U. Voigt, G. Pedrizzetti, and P. Lysyansky et al., "Definitions for a common standard for 2D speckle tracking echocardiography. consensus document of the EACVI/ASE/industry task force to standardize deformation imaging," European Heart Journal, 2014, accepted.

[74] N. Toussaint, C. T. Stoeck, T. Schaeffter, S. Kozerke, M. Sermesant, and P. G. Batchelor, "In vivo human cardiac fibre architecture estimation using shape-based diffusion tensor processing," Medical Image Analysis, vol. 17 , no. 8 , pp. $1243-1255,2013$

[75] K. Lekadir, C. Hoogendoorn, M. Pereanez, X. Alba, A. Pashaei, and A. Frangi, "Statistical personalization of ventricular fiber orientation using shape predictors," Medical Imaging, IEEE Transactions on, vol. 33, no. 4, pp. 882-890, April 2014.

[76] T. Edvardsen, B. L. Gerber, J. Garot, D. A. Bluemke, J. A. Lima, and O. A. Smiseth, "Quantitative assessment of intrinsic regional myocardial deformation by doppler strain rate echocardiography in humans validation against three-dimensional tagged magnetic resonance imaging," Circulation, vol. 106, pp. 50-56, 2002.

[77] C. Compas, E. Wong, X. Huang, S. Sampath, B. Lin, P. Pal, X. Papademetris, K. Thiele, D. Dione, M. Stacy, L. Staib, A. Sinusas, M. O'Donnell, and J. Duncan, "Radial basis functions for combining shape and speckle tracking in 4d echocardiography," Medical Imaging, IEEE Transactions on, vol. 33, no. 6, pp. 1275-1289, June 2014.

[78] J. N. Cohn, R. Ferrari, and N. Sharpe, "Cardiac remodelingconcepts and clinical implications: a consensus paper from an international forum on cardiac remodeling," Journal of the American College of Cardiology, vol. 35 , no. 3, pp. $569-582,2000$.

[79] C. Butakoff, S. Balocco, S. Ordas, and A. Frangi, "Simulated 3D ultrasound LV cardiac images for active shape model training," in Proc. of SPIE, vol. 6512, 2007, pp. 65 123U-8.

[80] A. Prakosa, M. Sermesant, H. Delingette, E. Saloux, P. Allain, P. Cathier, P. Etyngier, N. Villain, and N. Ayache, "Non-invasive activation times estimation using 3D echocardiography," in Statistical Atlases and Computational Models of the Heart, ser. Lecture Notes in Computer Science, O. Camara, M. Pop, K. Rhode, M. Sermesant, N. Smith, and A. Young, Eds. Springer Berlin Heidelberg, 2010, vol. 6364, pp. 212-221.

[81] A. Prakosa, M. Sermesant, P. Allain, N. Villain, C. Rinaldi, K. Rhode, R. Razavi, H. Delingette, and N. Ayache, "Cardiac electrophysiological activation pattern estimation from images using a patient-specific database of synthetic image sequences," Biomedical Engineering, IEEE Transactions on, vol. 61, no. 2, pp. 235-245, Feb 2014.

[82] T. Blum, A. Rieger, N. Navab, H. Friess, and M. Martignoni, "A review of computer-based simulators for ultrasound training," Simul Healthc., vol. 8, no. 2, pp. 98-108, Apr. 2012.

[83] M. Alessandrini, B. Heyde, S. Cygan, M. Sermesant, H. Delingette, O. Bernard, M. De Craene, and J. D'hooge, "Elastic registration vs. block matching for quantification of cardiac function with 3D ultrasound: Initial results of a direct comparison in silico based on a new evaluation pipeline," in Ultrasonics Symposium (IUS), 2014 IEEE International, Sept 2014, pp. 608-611. 